Research Paper

\title{
Efficacy and safety of different interventions in castration resistant prostate cancer progressing after docetaxel-based chemotherapy: Bayesian network analysis of randomized controlled trials
}

\author{
Yue Zhao ${ }^{3^{*}}$, Hao Huang ${ }^{1 *}$, Changhao Chen ${ }^{1,2^{*}}$, Hao Liu ${ }^{1}$, Hongwei Liu ${ }^{1,2}$, Feng Su${ }^{1,2}$, Junming Bi ${ }^{1,2}$, Thomas \\ B. Lam ${ }^{4}$, Jiaping $\mathrm{Li}^{3^{*}}$, Tianxin Lin ${ }^{1,2 \varpi}$, Jian Huang ${ }^{1 凶}$ \\ 1. Department of Urology, Sun Yat-Sen Memorial Hospital of Sun Yat-sen University, Guangzhou, China; \\ 2. Guangdong Provincial Key Laboratory of Malignant Tumor Epigenetics and Gene Regulation, Sun Yat-sen Memorial Hospital of Sun Yat-sen University, \\ Guangzhou, China; \\ 3. Department of Interventional oncology, First Affiliated Hospital of Sun Yat-Sen University, Guangzhou, China. \\ 4. Academic Urology Unit, University of Aberdeen, Cornhill Road, Aberdeen, UK. \\ "These authors (Changhao Chen, Hao Huang and Yue Zhao) contributed equally to this study.
}

$\triangle$ Corresponding authors: Jian Huang MD, PhD. Department of Urology, Sun Yat-Sen Memorial Hospital, Sun Yat-Sen University, 107 Yan-Jiang Xi Road, Guangzhou, 510120, China. Tel. +86 20 81332603; Fax: +86 20 81332853. E-mail address: changhaochen526@gmail.com and Tianxin Lin MD, PhD. Department of Urology, Sun Yat-Sen Memorial Hospital, Sun Yat-Sen University, 107 Yan-Jiang Xi Road, Guangzhou, 510120, China. Tel. +86 20 81332603; Fax: +86 2081332853.

E-mail address: tianxinl@sina.com

(c) Ivyspring International Publisher. This is an open access article distributed under the terms of the Creative Commons Attribution (CC BY-NC) license (https:// creativecommons.org/licenses/by-nc/4.0/). See http://ivyspring.com/terms for full terms and conditions.

Received: 2017.08.14; Accepted: 2017.12.08; Published: 2018.01.11

\begin{abstract}
Background: Most patients receiving docetaxel-based chemotherapy for castration resistant prostate cancer (CRPC) will eventually progress, and the optimal interventions for these patients are controversial. The objective of our study is to evaluate the clinical efficacy and safety of pharmacological interventions for CRPC patients progressing after docetaxel-based chemotherapy.

Methods: A systematic review and Bayesian network meta-analysis of the literature was carried out according to standard methods. Major electronic databases including PubMed, Web of Science and Embase were searched until Jan 2017. Hazard ratios (HRs) and odds ratios (ORs) with corresponding $95 \%$ credible intervals (Crls) were used to estimate the association.

Results: 17 Randomized Controlled Trials (RCTs) comprising 14 different interventions with 12347 patients were enrolled. Compared with control arms, Abiraterone Acetate (HR: 0.70, 95\%Crl: 0.63-0.79), Cabazitaxel (HR: 0.70, 95\%Crl: 0.51-0.95) and Enzalutamide (HR: 0.63, 95\%Crl: 0.53-0.75) presented similar benefits in term of OS. Enzalutamide showed superiority over PFS and PSA response with a highest probability to rank 1. Moreover, sensitivity analysis showed that Abiraterone Acetate (HR: 0.71, 95\%Crl: 0.63-0.78) exhibited the most efficacious intervention of being rank 1 in term of OS compared with control arms, followed by Cabazitaxel and Cetuximab. On the other hand, Abiraterone Acetate (OR: 0.86, 95\%Crl: 0.35-2.03) presented no significant toxicities compared with control arms.

Conclusions: Our results demonstrated that Abiraterone Acetate might be the optimal intervention for CRPC patients after docetaxel failure with acceptable tolerability. Future well-designed RCTs and systematic reviews are needed to validate these findings.
\end{abstract}

Key words: Castration resistant prostate cancer; Pharmacological Interventions; Docetaxel-based Chemotherapy; Bayesian network meta-analysis; Abiraterone Acetate; Enzalutamide

\section{Introduction}

Prostate cancer (PCa) is the second most commonly diagnosed cancer in the world, with
161,360 estimated new cases of PCa in 201712 . Less than $5 \%$ of patients present with metastatic disease, 
up to $40 \%$ of detected cases will eventually develop metastasis ${ }^{3}$. Androgen deprivation therapy (ADT) has been the standard of care for metastatic PCa ${ }^{4-6}$. However, most patients eventually stop responding to ADT and are categorized as castration resistant prostate cancer $(\mathrm{CRPC})^{7}$, which is defined as either biochemically or clinically progressive metastatic disease despite castrate serum levels of testosterone $(<50 \mathrm{ng} / \mathrm{dL} ;<1.7 \mathrm{nmol} / \mathrm{L})^{8}$. After developing mCRPC, it is dismal with a median survival of 12 to 18 months .

Docetaxel is a standard first-line chemotherapy in men with CRPC based on improvements in overall survival (OS) and progression free survival (PFS), compared with Mitoxantrone plus prednisone 1011. However, most of patients who receive docetaxel-based chemotherapy for MCRPC will eventually progress, and no consensus exist for the optimal interventions after docetaxel failure. The decision to initiate therapy demand the available high-level evidence of efficacy and tolerability in the post-docetaxel CRPC setting. Treatment options include Abiraterone with prednisone ${ }^{12}$, Enzalutamide 13, Radium-22314, Cabazitaxel ${ }^{15}$ and so on. However, there are few randomized controlled trials (RCT) comparing different treatment strategies to inform patients regarding the comparative effectiveness of those interventions ${ }^{16-18}$. Therefore, pair-wise metaanalysis couldn't generate clear hierarchies among available treatments in this case, because they provide only partial information, and hence, do not optimally inform decision-making.

Network meta-analysis has been recently demonstrated to compare different interventions and integrate evidence from direct comparisons and indirect comparisons across a network of RCTs ${ }^{19}{ }^{20}$. Moreover, Bayesian network meta-analysis which synthesizes all evidence on the relative treatment effects, enables unified and coherent analysis of relevant RCTs ${ }^{21}$. Therefore, we applied the established methodology in the comprehensive network involving post-docetaxel treatments evaluating the clinical efficacy and tolerability of interventions for CRPC patients.

\section{Methods}

This systematic review was performed according to the Preferred Reporting Items for Systematic Reviews and Meta-analyses (PRISMA) guidelines 2223.

\section{Search strategy and selection criteria}

We identified all relevant RCTs published from inception up to January 30, 2017 for assessing the clinical significance of currently available interventions for CRPC patients after docetaxel failure from the following databases, including PubMed, EMBASE, MEDLINE, Web of Science, the Cochrane Library, Central Register of Controlled Trials. The $\mathrm{MeSH}$ terms were correctly adjusted in different database. The search strategy of PubMed is as follows: (("prostatic neoplasms"[MeSH Terms] OR ("prostatic" [All Fields] AND "neoplasms"[All Fields]) OR "prostatic neoplasms"[All Fields] OR ("prostate"[All Fields] AND "cancer"[All Fields]) OR "PCa"[All Fields]) AND ("orchiectomy"[MeSH Terms] OR "orchiectomy"[All Fields] OR "castration"[All Fields] OR "castration"[MeSH Terms]) AND resistant[All Fields] AND ("docetaxel"[MeSH Terms] OR "docetaxel-based chemotherapy" [All Fields] OR "taxane-based chemotherapy" [All Fields]) AND ("resistant" [All Fields] OR "failure" [All Fields] OR "refractory" [All Fields]) AND ("clinical trial" [Publication Type] OR "clinical trials as topic"[MeSH Terms] OR "clinical trial"[All Fields]). No language restrictions were applied. We also contacted the corresponding authors to acquire information if more information was needed.

Eligible studies in this network meta-analysis were RCTs that met the following inclusion criteria: (a) Patients: Histologically confirmed prostate cancer aged $\geq 18$ years with castrate levels of serum testosterone $(<50 \mathrm{ng} / \mathrm{dL})$ were eligible if they had failed previous docetaxel-containing chemotherapy; documented progression was based on PSAWG criteria or radiographic progression in soft tissue or bone; (b) Intervention: established therapies for management of CRPC patients after docetaxel failure including chemotherapy, immunotherapy, androgen receptor targeting etc.; (c) Comparator: another active agent, Prednisone plus placebo, placebo, or no intervention; (d) Outcome: OS, PFS, PSA response and adverse events. We excluded observational studies, and trials comparing different doses of the same medication without an alternative intervention/comparator arm.

\section{Data Extraction and quality assessment}

Two authors (C.H. Chen and Y. Zhao) screened all the titles and abstracts identified by the search strategy independently, the results were assessed for eligibility. Disagreement was resolved by consensus between two authors or by a senior author (J. Huang). Data collection form was designed to collect information from these publications including first author, year of publication, follow-up, study type, participants, intervention and outcomes. We considered the OS, PFS and adverse events (Grade 3-4) for our primary analyses. Secondary outcome included PSA response (proportion of patients achieving $\geq 50 \%$ PSA decline according to PSAWG 
criteria). Since randomized trials used low-dose oral daily corticosteroids in combination with mitoxantrone 24, they have been employed in combination with taxanes, with the rationale being to maintain balance between the arms ${ }^{15}{ }^{25}$. However, the impact of prednisone on survival, as a single agent or in combination, remains unclear. Recently, comprehensive studies estimating the efficacy and safety with the use of daily prednisone indicated that Prednisone plus placebo arm showed similar benefit compared with placebo 2627 . Thus, the reference standard was regarded as Prednisone plus placebo in the present study. For studies presenting the same RCT, we extracted the updated data for our meta-analysis, such as TROPIC trial reported by Bahl et al $(2013)^{28}$ and de Bono et al $(2010)^{15}$. In order to evaluate the quality of including studies in this network meta-analysis, we assessed the risk of bias using the Cochrane Collaboration's Risk of Bias tool ${ }^{29}$.

\section{Statistical Analysis}

We fitted a Bayesian network meta-analysis model for each outcome separately, combining direct evidence for each comparison with indirect evidence, for all pair-wise comparisons simultaneously. We evaluated inconsistency by comparing the estimates from direct comparisons and those from indirect comparisons for magnitude and direction of the point estimates. We estimated treatment effects by posterior means with corresponding $95 \%$ credible intervals (CrIs) and adjusted for different arms. Both the fixed and random effects models were applied. The differences between the two models were that the latter considers between-study variance, thereby producing wider CrIs, and was preferred in the presence of heterogeneity. Bayesian deviance information criterion (DIC) statistics were utilized to compare the two models. The DIC statistics provide a model fit measure which penalizes model complexity with lower values. We updated Markov chain Monte Carlo model with 100,000 simulated draws after a burn in of 10,000 iterations. The probability of each treatment being the best, second best, third best and so on, from the rank orderings of the treatments at each iteration of the Markov chain were recorded ${ }^{30}$. The network meta-analyses were built in WinBUGS (MRC Biostatistics Unit, Cambridge, UK) 20.

Pair-wise meta-analyses were conducted on endpoints. The traditional direct meta-analysis, two or more studies that compared two interventions of interest were statistically combined. The survival endpoints were expressed as hazard ratio (HR). Estimated survival curves for OS and PFS were plotted using the method described by Parmar et $\mathrm{al}^{31}$.
The estimated hazard ratios of OS $32-35$ and PFS 3233 were obtained by using the above method. Dichotomous variables for PSA response and adverse events were presented as odds ratio (OR) with $95 \%$ confidence intervals (CIs). A two-sided $p$ value of less than 0.05 was considered significant. The pair-wise meta-analysis was performed using Stata 13.0 (StataCorp, College Station, TX, USA).

Sensitivity analyses of the primary outcome would be applied in the RCTs which presented high-quality with control arm of Prednisone plus placebo. The threshold for good quality studies for sensitivity analysis was based on the Cochrane assessment tool for assessing the risk of bias. The studies with low risk of selection and flow bias were included. Publication bias was assessed by examining funnel plot symmetry and Egger's regression test ${ }^{36}$.

\section{Results}

\section{Search Results}

From a total of 922 unique studies comparing all dfferent interventions identified using the search strategy, 17 RCTs composing of 14 interventions met the eligibility criteria for the current study. A flow chart of trial selection was shown in Figure 1. Eight RCTs of CRPC therapies were excluded because of unclear primary outcome assessment 37-41 and comparison of different dosing regimens without a common comparator group 42-44. One study ${ }^{45}$ presenting Mitoxantrone plus prednisone was excluded because it did not meet our inclusion criterion for data integration of control arms.

Table 1 summarized the main characteristics of RCTs included in the network meta-analysis. Overall, 12347 mCRPC patients with docetaxel failure were randomly enrolled to included studies. Median follow-up was 21 (range 12.8-36 months) and the sample size ranged from 82 to 1199 people, with a median sample size of 755 . Based on the interventions under comparison, the included trials were classified into the following five categories, 1) Chemotherapy: Satraplatin, Cabazitaxel, Ixabepilone, Mitoxantrone; 2) Hormonal Strategies: Abiraterone acetate, Enzalutamide, Orteronel; 3) Target therapies: Sunitinib, Cetuximab, Rilotumumab, Cabozantinib; 4) Immune strategies: Siltuximab; 5) Bone-targeting agents: Ra-223. 17 trials provided OS, of which 11 trials reported PFS and 15 trials presented adverse events as primary endpoints for comparisons. 14 trials showed PSA response as secondary endpoint for comparisons. 
Table 1. Characteristics of the studies included in the network meta-analysis

\begin{tabular}{|c|c|c|c|c|c|c|}
\hline $\begin{array}{l}\text { Study, Year of } \\
\text { Publication; } \\
\text { Identifier }\end{array}$ & $\begin{array}{l}\text { Country; } \\
\text { Inclusion Period }\end{array}$ & $\begin{array}{l}\text { Follow-up median } \\
\text { month, }(95 \% \mathrm{CI})\end{array}$ & Study type & Participants & Intervention & Outcomes \\
\hline Bahl et al (2013) & $\begin{array}{l}\text { Multi-center, } \\
\text { Jan } \\
\text { 2007-September } \\
2009\end{array}$ & $25.5(20.7-30.0)$ & $\begin{array}{l}\text { Phase III, } \\
\text { Open label, } \\
\text { RCT }\end{array}$ & $\begin{array}{l}\text { 1) Pathologically proven prostate cancer; } \\
\text { 2) Surgical or hormone-induced castration; } \\
\text { 3) Disease progression; 4) docetaxel failure }\end{array}$ & $\begin{array}{l}\text { Experimental: Cabazitaxel + } \\
\text { Prednisone } \\
\text { Control: Mitoxantrone }+ \\
\text { Prednisone }\end{array}$ & OS, \\
\hline $\begin{array}{l}\text { de Bono et al } \\
(2010)\end{array}$ & $\begin{array}{l}\text { Multi-center, } \\
\text { Jan 2007-Oct } \\
2008\end{array}$ & $12.8(7.8-16.9)$ & $\begin{array}{l}\text { Phase III, } \\
\text { Open label, } \\
\text { RCT }\end{array}$ & $\begin{array}{l}\text { 1) Pathologically proven prostate cancer; } \\
\text { 2) Surgical or hormone-induced castration; } \\
\text { 3) Disease progression; 4) docetaxel failure }\end{array}$ & $\begin{array}{l}\text { Experimental: Cabazitaxel + } \\
\text { Prednisone } \\
\text { Control: Mitoxantrone }+ \\
\text { Prednisone }\end{array}$ & $\begin{array}{l}\text { OS, PFS, } \\
\text { PSA } \\
\text { Response, } \\
\text { Adverse } \\
\text { event }\end{array}$ \\
\hline $\begin{array}{l}\text { de Bono et al } \\
(2011)\end{array}$ & $\begin{array}{l}\text { Multi-center, } \\
\text { May 2008-Oct } \\
2012\end{array}$ & 12.8 & $\begin{array}{l}\text { Phase III, } \\
\text { Double blind, } \\
\text { RCT }\end{array}$ & $\begin{array}{l}\text { 1) Pathologically proven } \\
\text { prostate cancer; 2) Surgical or } \\
\text { hormone-induced } \\
\text { castration; 3) Disease } \\
\text { progression; 4) Metastasis; } \\
\text { 5) docetaxel failure }\end{array}$ & $\begin{array}{l}\text { Experimental: Abiraterone } \\
\text { acetate +Prednisone } \\
\text { Control: Prednisone }+ \\
\text { Placebo }\end{array}$ & $\begin{array}{l}\text { OS, PFS, } \\
\text { PSA } \\
\text { Response, } \\
\text { Adverse } \\
\text { event }\end{array}$ \\
\hline $\begin{array}{l}\text { Fizazi et al (1) } \\
(2012)\end{array}$ & $\begin{array}{l}\text { Multi-center, } \\
\text { Nov 2006-Nov } \\
2008\end{array}$ & NA & $\begin{array}{l}\text { Phase II, } \\
\text { Open label, } \\
\text { RCT }\end{array}$ & $\begin{array}{l}\text { 1) Pathologically proven } \\
\text { prostate cancer; 2) Surgical or } \\
\text { hormone-induced } \\
\text { castration; 3) Disease } \\
\text { progression; 4) Metastasis; } \\
\text { 5) docetaxel failure }\end{array}$ & $\begin{array}{l}\text { Experimental: Siltuximab + } \\
\text { Mitoxantrone + Prednisone } \\
\text { Control: Mitoxantrone + } \\
\text { Prednisone }\end{array}$ & $\begin{array}{l}\text { OS, PFS, } \\
\text { PSA } \\
\text { Response, } \\
\text { Adverse } \\
\text { event }\end{array}$ \\
\hline $\begin{array}{l}\text { Fizazi et al (2) } \\
(2012)\end{array}$ & $\begin{array}{l}\text { Multi-center, } \\
\text { May 2008-Oct } \\
2012\end{array}$ & $20.2(18.4-22.1)$ & $\begin{array}{l}\text { Phase III, } \\
\text { Double blind, } \\
\text { RCT }\end{array}$ & $\begin{array}{l}\text { 1) Pathologically proven } \\
\text { prostate cancer; 2) Surgical or } \\
\text { hormone-induced } \\
\text { castration; 3) Disease } \\
\text { progression; 4) Metastasis; } \\
\text { 5) docetaxel failure }\end{array}$ & $\begin{array}{l}\text { Experimental: Abiraterone } \\
\text { acetate +Prednisone } \\
\text { Control: Prednisone }+ \\
\text { Placebo }\end{array}$ & $\begin{array}{l}\text { OS, } \\
\text { PSA } \\
\text { response, } \\
\text { Adverse } \\
\text { event }\end{array}$ \\
\hline Fizazi et al (2015) & $\begin{array}{l}\text { Multi-center, } \\
\text { Nov 2010-Sep } \\
2014\end{array}$ & NA & $\begin{array}{l}\text { Phase III, } \\
\text { Open label, } \\
\text { RCT }\end{array}$ & $\begin{array}{l}\text { 1) Pathologically proven } \\
\text { prostate cancer; 2) Surgical or } \\
\text { hormone-induced } \\
\text { castration; 3) Disease } \\
\text { progression; 4) Metastasis; } \\
\text { 5) docetaxel failure }\end{array}$ & $\begin{array}{l}\text { Experimental: Orteronel + } \\
\text { Prednisone } \\
\text { Control: Prednisone }+ \\
\text { Placebo }\end{array}$ & $\begin{array}{l}\text { OS, PFS, } \\
\text { PSA } \\
\text { Response, } \\
\text { Adverse } \\
\text { event }\end{array}$ \\
\hline $\begin{array}{l}\text { Fleming et al } \\
(2012)\end{array}$ & $\begin{array}{l}\text { Multi-center, } \\
\text { May 2008-June } \\
2011\end{array}$ & 30 & $\begin{array}{l}\text { Phase II, } \\
\text { Open label, } \\
\text { RCT }\end{array}$ & $\begin{array}{l}\text { 1) Pathologically proven } \\
\text { prostate cancer; 2) Surgical or } \\
\text { hormone-induced } \\
\text { castration; 3) Disease } \\
\text { progression; 4) Metastasis; } \\
\text { 5) docetaxel failure }\end{array}$ & $\begin{array}{l}\text { Experimental: Cetuximab + } \\
\text { Mitoxantrone + Prednisone } \\
\text { Control: Mitoxantrone }+ \\
\text { Prednisone }\end{array}$ & $\begin{array}{l}\text { PFS, OS, } \\
\text { PSA } \\
\text { Response } \\
\text { Adverse } \\
\text { event }\end{array}$ \\
\hline $\begin{array}{l}\text { Hoskin et al } \\
(2014)\end{array}$ & $\begin{array}{l}\text { Multi-center, } \\
\text { June 2008- Feb } \\
2014\end{array}$ & NA & $\begin{array}{l}\text { Phase III, } \\
\text { Double blind, } \\
\text { RCT }\end{array}$ & $\begin{array}{l}\text { 1) Pathologically proven } \\
\text { prostate cancer; 2) Surgical or } \\
\text { hormone-induced } \\
\text { castration; 3) Disease } \\
\text { progression; 4) Bone } \\
\text { metastasis; } \\
\text { 5) docetaxel failure }\end{array}$ & $\begin{array}{l}\text { Experimental: Radium-223 } \\
\text { Control: Placebo }\end{array}$ & $\begin{array}{l}\text { OS, } \\
\text { Adverse } \\
\text { event }\end{array}$ \\
\hline $\begin{array}{l}\text { Kantoff et al } \\
(1999)\end{array}$ & $\begin{array}{l}\text { Multi-center, } \\
\text { Oct 1992-Sep } \\
1995\end{array}$ & NA & RCT & $\begin{array}{l}\text { 1) Pathologically proven prostate cancer; } \\
\text { 2) Surgical or hormone-induced castration; } \\
\text { 3) Disease progression; 4) docetaxel failure }\end{array}$ & $\begin{array}{l}\text { Experimental: } \\
\text { Mitoxantrone + Prednisone } \\
\text { Control: Prednisone }\end{array}$ & $\begin{array}{l}\text { OS, PFS, } \\
\text { PSA } \\
\text { Response, } \\
\text { Adverse } \\
\text { event }\end{array}$ \\
\hline $\begin{array}{l}\text { Michaelson et al } \\
\text { (2014) }\end{array}$ & $\begin{array}{l}\text { Multi-center, } \\
\text { Jul 2008-Aug } \\
2010\end{array}$ & NA & $\begin{array}{l}\text { Phase III, } \\
\text { Double blind, } \\
\text { RCT }\end{array}$ & $\begin{array}{l}\text { 1) Pathologically proven } \\
\text { prostate cancer; 2) Surgical or } \\
\text { hormone-induced } \\
\text { castration; 3) Disease } \\
\text { progression; 4) Metastasis; } \\
\text { 5) docetaxel failure }\end{array}$ & $\begin{array}{l}\text { Experimental: Sunitinib + } \\
\text { Prednisone } \\
\text { Control: Prednisone } \\
+ \text { Placebo }\end{array}$ & $\begin{array}{l}\text { OS, PFS, } \\
\text { PSA } \\
\text { Response, } \\
\text { Adverse } \\
\text { event }\end{array}$ \\
\hline Parker et al (2013) & $\begin{array}{l}\text { Multi-center, } \\
\text { Jan 2008-Feb } \\
2011\end{array}$ & 36 & $\begin{array}{l}\text { Phase III, } \\
\text { Double blind, } \\
\text { RCT }\end{array}$ & $\begin{array}{l}\text { 1) Pathologically proven } \\
\text { prostate cancer; 2) Surgical or } \\
\text { hormone-induced } \\
\text { castration; 3) Disease } \\
\text { progression; 4) Bone } \\
\text { metastasis; } \\
\text { 5) docetaxel failure }\end{array}$ & $\begin{array}{l}\text { Experimental: Radium-223 } \\
\text { Control: Placebo }\end{array}$ & $\begin{array}{l}\text { OS, } \\
\text { Adverse } \\
\text { event }\end{array}$ \\
\hline $\begin{array}{l}\text { Rosenberg et al } \\
(2007)\end{array}$ & $\begin{array}{l}\text { Multi-center, } \\
\text { Feb 2003-Jun } \\
2005\end{array}$ & NA & $\begin{array}{l}\text { Phase II, } \\
\text { RCT }\end{array}$ & $\begin{array}{l}\text { 1) Pathologically proven prostate cancer; } \\
\text { 2) Surgical or hormone-induced castration; } \\
\text { 3) Disease progression; 4) docetaxel failure }\end{array}$ & $\begin{array}{l}\text { Experimental: Ixabepilone } \\
\text { Control: Mitoxantrone }+ \\
\text { Prednisone }\end{array}$ & $\begin{array}{l}\text { OS, } \\
\text { PSA } \\
\text { Response, } \\
\text { Adverse } \\
\text { event }\end{array}$ \\
\hline Ryan et al (2012) & $\begin{array}{l}\text { Multi-center, } \\
\text { Mar 2009-Dec } \\
2009\end{array}$ & 21 & $\begin{array}{l}\text { Phase II, } \\
\text { Double blind, } \\
\text { RCT }\end{array}$ & $\begin{array}{l}\text { 1) Pathologically proven } \\
\text { prostate cancer; } 2 \text { ) Surgical or } \\
\text { hormone-induced }\end{array}$ & $\begin{array}{l}\text { Experimental One: } \\
\text { Rilotumumab }(15 \mathrm{mg} / \mathrm{kg})+ \\
\text { Mitoxantrone + Prednisone }\end{array}$ & $\begin{array}{l}\text { OS, PFS, } \\
\text { PSA } \\
\text { response, }\end{array}$ \\
\hline
\end{tabular}




\begin{tabular}{|c|c|c|c|c|c|c|}
\hline $\begin{array}{l}\text { Study, Year of } \\
\text { Publication; } \\
\text { Identifier }\end{array}$ & $\begin{array}{l}\text { Country; } \\
\text { Inclusion Period }\end{array}$ & $\begin{array}{l}\text { Follow-up median } \\
\text { month, }(95 \% \mathrm{CI})\end{array}$ & Study type & Participants & Intervention & Outcomes \\
\hline & & & & $\begin{array}{l}\text { castration; 3) Disease } \\
\text { progression; 4) Metastasis; } \\
\text { 5) docetaxel failure }\end{array}$ & $\begin{array}{l}\text { Experimental Two: } \\
\text { Rilotumumab }(7.5 \mathrm{mg} / \mathrm{kg})+ \\
\text { Mitoxantrone + Prednisone } \\
\text { Control: Mitoxantrone + } \\
\text { Prednisone + Placebo }\end{array}$ & $\begin{array}{l}\text { Adverse } \\
\text { event }\end{array}$ \\
\hline Scher et al (2012) & $\begin{array}{l}\text { Multi-center, } \\
\text { Sep 2009-Nov } \\
2010\end{array}$ & NA & $\begin{array}{l}\text { Phase III, } \\
\text { Double blind, } \\
\text { RCT }\end{array}$ & $\begin{array}{l}\text { 1) Pathologically proven prostate cancer; } \\
\text { 2) Surgical } \\
\text { or hormone-induced } \\
\text { castration; 3) Disease } \\
\text { progression; 4) docetaxel } \\
\text { failure }\end{array}$ & $\begin{array}{l}\text { Experimental: } \\
\text { Enzalutamide } \\
\text { Control: Placebo }\end{array}$ & $\begin{array}{l}\text { OS, PFS, } \\
\text { PSA } \\
\text { Response, } \\
\text { Adverse } \\
\text { event }\end{array}$ \\
\hline Smith et al (2016) & $\begin{array}{l}\text { Multi-center, } \\
\text { Jul 2012-Nov } \\
2014\end{array}$ & NA & $\begin{array}{l}\text { Phase III, } \\
\text { Double blind, } \\
\text { RCT }\end{array}$ & $\begin{array}{l}\text { 1) Pathologically proven } \\
\text { prostate cancer; 2) Surgical or } \\
\text { hormone-induced } \\
\text { castration; 3) Disease } \\
\text { progression; 4) Bone } \\
\text { metastasis; } \\
\text { 5) docetaxel failure }\end{array}$ & $\begin{array}{l}\text { Experimental: cabozantinib } \\
\text { Control: Prednisone }\end{array}$ & $\begin{array}{l}\text { OS, PFS } \\
\text { PSA } \\
\text { Response }\end{array}$ \\
\hline $\begin{array}{l}\text { Sternberg et al } \\
(2009)\end{array}$ & $\begin{array}{l}\text { Multi-center, } \\
\text { Sep 2003-Jan } \\
2006\end{array}$ & NA & $\begin{array}{l}\text { Phase III, } \\
\text { Double blind, } \\
\text { RCT }\end{array}$ & $\begin{array}{l}\text { 1) Pathologically proven } \\
\text { prostate cancer; 2) Surgical or } \\
\text { hormone-induced } \\
\text { castration; 3) Disease } \\
\text { progression; 4) Bone } \\
\text { metastasis; } \\
\text { 5) docetaxel failure }\end{array}$ & $\begin{array}{l}\text { Experimental: Satraplatin }+ \\
\text { Prednisone } \\
\text { Control: Prednisone }+ \\
\text { Placebo }\end{array}$ & $\begin{array}{l}\text { OS, PFS, } \\
\text { PSA } \\
\text { response, } \\
\text { Adverse } \\
\text { event }\end{array}$ \\
\hline Sun et al (2016) & $\begin{array}{l}\text { Multi-center, } \\
\text { Aug 2012-June } \\
2014\end{array}$ & NA & $\begin{array}{l}\text { Phase III, } \\
\text { Double blind, } \\
\text { RCT }\end{array}$ & $\begin{array}{l}\text { 1) Pathologically proven prostate cancer; } \\
\text { 2) Surgical } \\
\text { or hormone-induced } \\
\text { castration; 3) Disease } \\
\text { progression; 4) docetaxel } \\
\text { failure }\end{array}$ & $\begin{array}{l}\text { Experimental: Abiraterone } \\
\text { acetate }+ \text { prednisone } \\
\text { Control: Prednisone }+ \\
\text { Placebo }\end{array}$ & $\begin{array}{l}\text { OS, } \\
\text { PSA } \\
\text { Response, } \\
\text { Adverse } \\
\text { event }\end{array}$ \\
\hline
\end{tabular}

NA: not applicable; RCT: randomized controlled trial; OS: overall survival; PFS: progression free survival

\section{Network meta-analysis outcomes}

We established a network meta-analysis to compare the OS, PFS, PSA response and adverse event of different interventions. The respective sets of HRs and ORs with the corresponding 95\% CrIs from the fixed effect model and random effect model had good consistency. The DIC values were lower in fixed effect model compared with random effect model for endpoints (range 5.36-10.07 vs range 5.57-88.68), indicating the fixed effect model had a substantially better fit than random effect model. Therefore, we applied the fixed effects model for the rest of the study. Supplementary Table 1 showed the summary of primary and secondary endpoints findings.

\section{Efficacy results}

A total number of 17 studies reported information of OS and 11 studies reported PFS were included in the present study. Figure 2 showed the full network diagram of eligible comparisons for OS, PFS and PSA response. In terms of OS, Abiraterone Acetate (HR: 0.70, 95\%CrI: 0.63-0.79), Enzalutamide (HR: 0.63, 95\%CrI: 0.53-0.75) and Cabazitaxel (HR: 0.70, 95\% CrI: 0.51-0.95) were superior to control arms. Moreover, as hormonal strategies, both Abiraterone Acetate and Enzalutamide were significantly increasing OS than other interventions (Figure 3). In terms of PFS, Enzalutamide (HR: 0.40, 95\%CrI: 0.35-0.46) was the most efficacious intervention with $87.9 \%$ cumulative probabilities of being rank 1 compared with control, followed by Cabozantinib and Abiraterone Acetate (Figure 4). In regards to PSA response, Abiraterone Acetate (OR: 6.25, 95\% CrI: 2.27-20.0) and Enzalutamide (OR: 50.0, 95\%CrI: 12.5-100.0) demonstrated significant benefits in terms of PSA response efficacy (Supplementary Figure 1).

\section{Safety results}

The network meta-analysis for adverse events (grade 3-4) was shown in Figure 3 and 4. Abiraterone Acetate (OR: 0.86, 95\%CrI: 0.35-2.03) and Enzalutamide (OR: 1.22, 95\%CrI: 0.31-5.60) presented no significant toxicities compared with control arms, whereas Mitoxantrone, Cabazitaxel, Ixabepilone, Cetuximab, Siltuximab and Rilotumumab were not as well tolerated compared with control arms for adverse events (grade 3-4).

\section{Pair-wise meta-analysis outcomes}

Results from pair-wise meta-analysis of including interventions were shown in Supplementary Figure 2, which were consistent with that of network meta-analysis. Pooled data showed that Abiraterone Acetate (HR: 0.70, 95\% CI: 0.62-0.77), Enzalutamide (HR: 0.63, 95\%CI: 0.52-0.74), 
Cabazitaxel (HR: 0.71, 95\%CI: 0.63-0.79) presented benefits compared with control in term of OS. Moreover, Abiraterone Acetate, Enzalutamide,
Cabazitaxel and Orteronel were associated with longer PFS and higher PSA response than control arms.
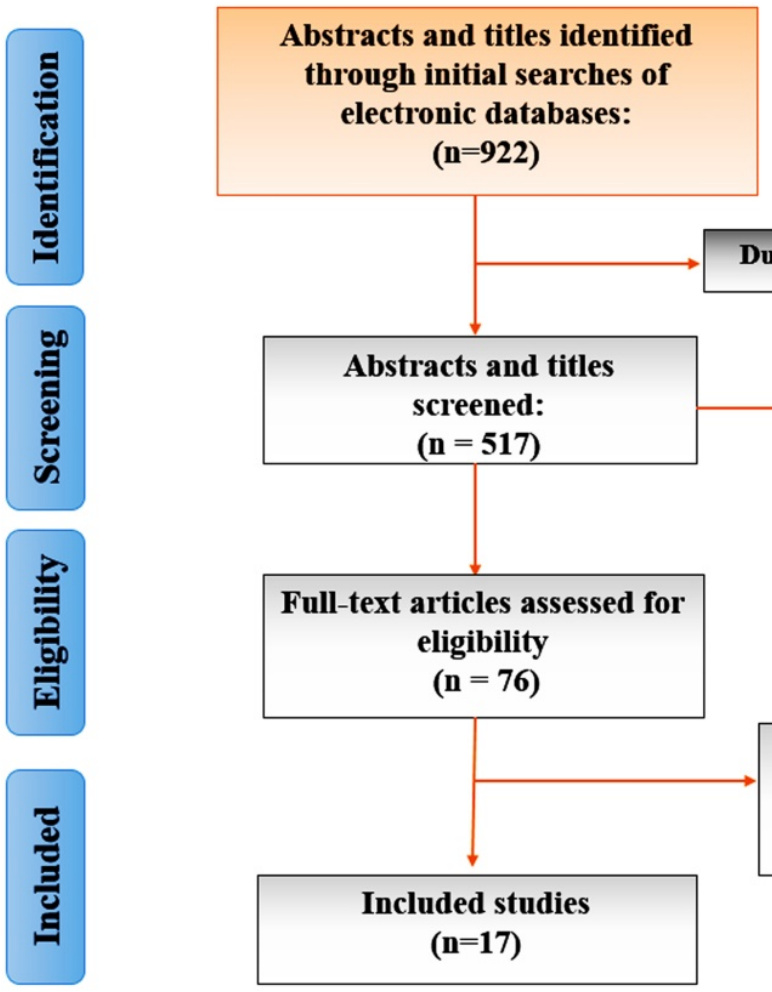

Excluded studies: $\mathbf{n}=\mathbf{5 9}$

-Not relevant to this review/other topic: $n=23$

-Not intra-patient comparison studies: $\mathbf{n}=\mathbf{2 0}$

-Not second-line treatment : $n=16$

Figure 1. The PRISMA flow chart of included studies in network meta-analysis.
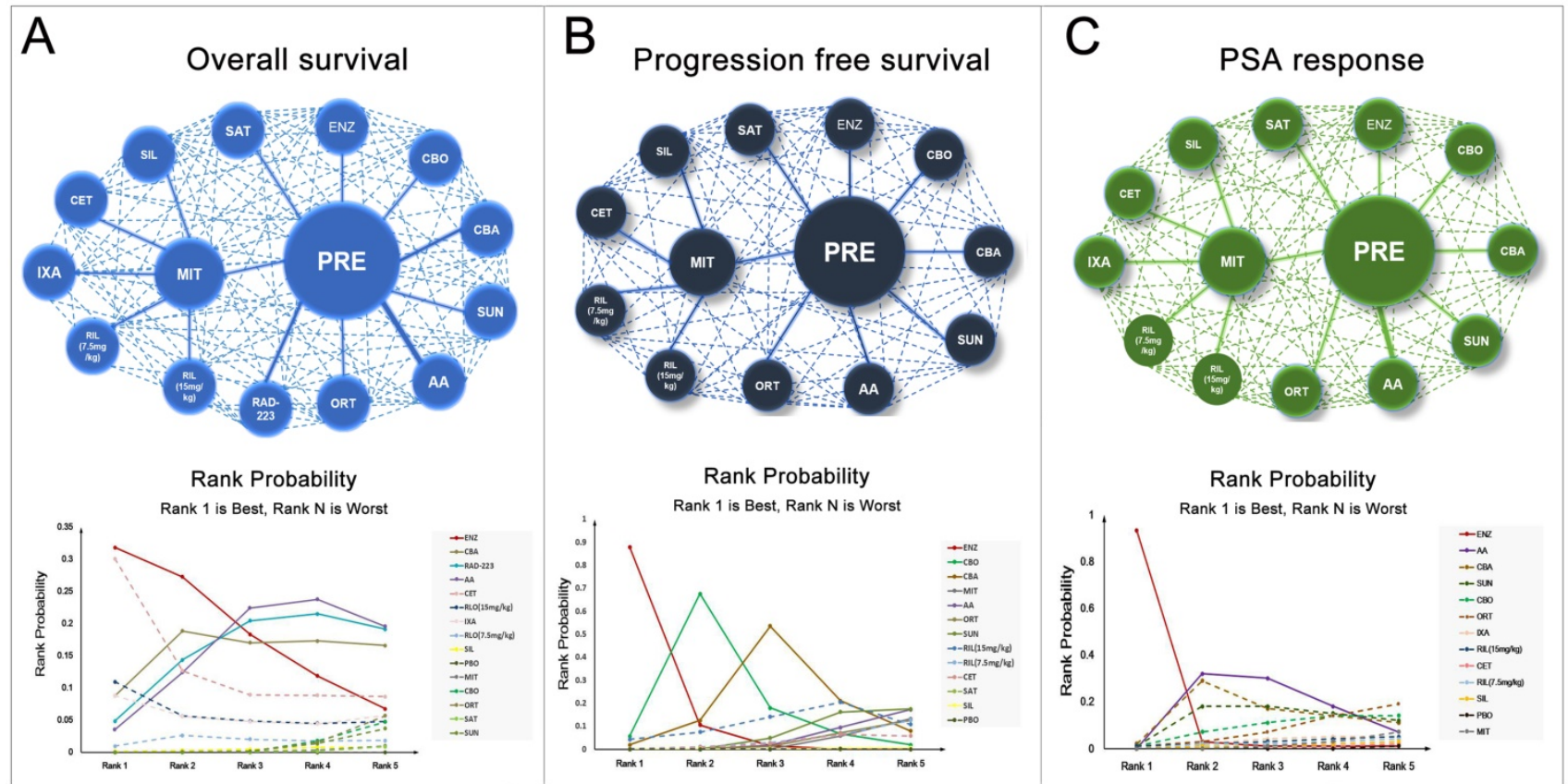

Figure 2. Network and rank probability of comparisons included in the analysis. Evidence network of different interventions for OS, PFS and PSA response for CRPC patients after docetaxel failure. The thickness of the connection line corresponds to the numbers of studies between comparators. Probabilities of each intervention ranking best, second, third, fourth and fifth best based on the fixed effects model. Full lines stand for agents with significant difference, while dash lines stand for agents without significant difference in comparisons. 


\begin{tabular}{|c|c|c|c|c|c|c|c|c|c|c|c|c|c|c|}
\hline MIT & $\begin{array}{c}\underline{0.09} \\
\underline{(0.02,0.55)}\end{array}$ & $\begin{array}{c}2.50 \\
(0.55,13.92)\end{array}$ & $\begin{array}{c}2.45 \\
(0.54,10.73)\end{array}$ & $\begin{array}{c}0.15 \\
(0.02,1.24)\end{array}$ & $\begin{array}{c}0.06 \\
\underline{0.01,0.49}\end{array}$ & $\begin{array}{c}0.68 \\
(0.13,3.19)\end{array}$ & $\begin{array}{c}0.14 \\
(0.02,1.16)\end{array}$ & $\begin{array}{c}\underline{0.06} \\
\underline{0.01,0.46)}\end{array}$ & $\begin{array}{c}0.08 \\
(0.02,0.34) \\
\end{array}$ & $\begin{array}{c}\underline{0.07} \\
\underline{(0.01,0.62)}\end{array}$ & $\begin{array}{c}1.07 \\
(0.22,4.85)\end{array}$ & $\begin{array}{c}0.16 \\
(0.02,1.24)\end{array}$ & $\begin{array}{c}1.89 \\
(0.41,9.20)\end{array}$ & $\begin{array}{c}1.89 \\
(0.41,9.20)\end{array}$ \\
\hline $\begin{array}{c}\frac{0.72}{(0.52,0.97)} \\
\underline{10.0}\end{array}$ & AA & $\begin{array}{c}\frac{28.32}{(2.71,309.42)} \\
(.75)\end{array}$ & $\begin{array}{c}\underline{26.97} \\
(2.79,284.16 \\
\end{array}$ & $\begin{array}{c}1.65 \\
(0.29,9.08)\end{array}$ & $\begin{array}{c}0.70 \\
(0.12,3.71)\end{array}$ & $\begin{array}{c}7.35 \\
(0.72,80.09)\end{array}$ & $\begin{array}{c}1.61 \\
(0.27,8.52)\end{array}$ & $\begin{array}{c}0.68 \\
(0.12,3.64)\end{array}$ & $\begin{array}{c}0.86 \\
(0.35,2.03)\end{array}$ & $\begin{array}{c}0.80 \\
(0.16,5.25)\end{array}$ & $\begin{array}{c}\underline{11.96} \\
\underline{1.12,134.91)}\end{array}$ & $\begin{array}{c}1.85 \\
(0.30,9.36)\end{array}$ & $\begin{array}{c}\underline{20.51} \\
(2.26 .239 .66)\end{array}$ & $\begin{array}{c}\underline{20.51} \\
(2.26,239.66 \\
\end{array}$ \\
\hline $\begin{array}{c}0.71 \\
(0.41,1.14)\end{array}$ & $\begin{array}{c}1.01 \\
(0.53,1.74)\end{array}$ & CET & $\begin{array}{c}0.97 \\
(0.09,8.06)\end{array}$ & $\begin{array}{c}\underline{0.06} \\
\underline{(0.01,0.75)} \\
\end{array}$ & $\begin{array}{c}\underline{0.02} \\
(\underline{0.01,0.29)}\end{array}$ & $\begin{array}{c}0.26 \\
(0.03,2.31)\end{array}$ & $\begin{array}{c}\underline{0.06} \\
\underline{(0.01,0.69)}\end{array}$ & $\begin{array}{c}\underline{0.02} \\
\underline{(0.01,0.29)}\end{array}$ & $\begin{array}{c}\underline{0.03} \\
(0.01,0.23) \\
\end{array}$ & $\begin{array}{c}\underline{0.03} \\
\underline{(0.01,0.36} \\
\end{array}$ & $\begin{array}{c}0.42 \\
(0.04,3.84)\end{array}$ & $\begin{array}{c}\underline{0.07} \\
\underline{(0.01,0.71)} \\
\end{array}$ & $\begin{array}{c}0.75 \\
(0.08,7.06)\end{array}$ & $\begin{array}{c}0.75 \\
(0.08,7.06)\end{array}$ \\
\hline 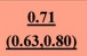 & $\begin{array}{c}1.01 \\
(0.71,1.39)\end{array}$ & $\begin{array}{c}1.05 \\
(0.65,1.68)\end{array}$ & CBA & $\begin{array}{c}\underline{0.06} \\
\underline{(0.01,0.88)}\end{array}$ & $\begin{array}{c}\underline{0.03} \\
\underline{(0.01,0.33)}\end{array}$ & $\begin{array}{c}4.46 \\
(0.31,61.70)\end{array}$ & $\begin{array}{c}\underline{0.06} \\
\underline{(0.01,0.79)}\end{array}$ & $\begin{array}{c}\underline{0.02} \\
(0.01,0.34)\end{array}$ & $\begin{array}{c}\underline{0.03} \\
(0.01,0.27) \\
\end{array}$ & $\begin{array}{c}\underline{0.03} \\
(\underline{0.01,0.44)}\end{array}$ & $\begin{array}{c}0.43 \\
(0.05,4.00)\end{array}$ & $\begin{array}{c}\underline{0.07} \\
(0.01,0.96)\end{array}$ & $\begin{array}{c}0.76 \\
(0.10,7.68)\end{array}$ & $\begin{array}{c}0.76 \\
(0.10,7.68)\end{array}$ \\
\hline $\begin{array}{c}0.93 \\
(0.64,1.27)\end{array}$ & $\begin{array}{c}\frac{1.29}{(1.05,1.56} \\
\underline{1.0}\end{array}$ & $\begin{array}{c}1.37 \\
(0.83 .2 .34)\end{array}$ & $\begin{array}{c}1.34 \\
(0.92,1.94)\end{array}$ & CBO & $\begin{array}{c}0.42 \\
(0.05,3.25)\end{array}$ & $\begin{array}{c}\underline{\mathbf{8 . 6 4}} \\
(\mathbf{1 . 0 3 , 7 7 . 2 3 )} \\
\end{array}$ & $\begin{array}{c}0.98 \\
(0.13,7.30)\end{array}$ & $\begin{array}{c}0.41 \\
(0.05,3.08)\end{array}$ & $\begin{array}{c}0.53 \\
(0.12,2.24)\end{array}$ & $\begin{array}{c}0.49 \\
(0.06,4.40)\end{array}$ & $\begin{array}{c}7.39 \\
(0.52,99.21)\end{array}$ & $\begin{array}{c}1.11 \\
(0.14,8.42)\end{array}$ & $\begin{array}{c}12.49 \\
(1.06,192.70) \\
\end{array}$ & $\begin{array}{c}12.49 \\
(1.06,192.70) \\
\end{array}$ \\
\hline $\begin{array}{c}0.65 \\
(0.45,0.9)\end{array}$ & $\begin{array}{c}0.90 \\
(0.73,1.11)\end{array}$ & $\begin{array}{c}0.96 \\
(0.58,1.64)\end{array}$ & $\begin{array}{c}0.94 \\
(0.65,1.36)\end{array}$ & $\begin{array}{c}\underline{0.7} \\
\underline{0.55,0.89)}\end{array}$ & ENZ & $\begin{array}{c}10.80 \\
(0.81,165.03)\end{array}$ & $\begin{array}{c}2.30 \\
(0.27,19.01)\end{array}$ & $\begin{array}{c}0.97 \\
(0.14,8.23)\end{array}$ & $\begin{array}{c}1.22 \\
(0.31,5.60)\end{array}$ & $\begin{array}{c}1.17 \\
(0.16,11.23)\end{array}$ & $\begin{array}{c}\frac{17.15}{(1.24,261.44)} \\
(1.0)\end{array}$ & $\begin{array}{c}2.64 \\
(0.33,20.47)\end{array}$ & $\begin{array}{c}29.43 \\
(2.51,478.27) \\
\end{array}$ & $\begin{array}{c}\frac{29.43}{(2.51,478.27} \\
\end{array}$ \\
\hline $\begin{array}{c}0.99 \\
(0.49,1.78)\end{array}$ & $\begin{array}{c}1.41 \\
(0.63,2.68)\end{array}$ & $\begin{array}{c}1.46 \\
(0.61,3.00)\end{array}$ & $\begin{array}{c}1.40 \\
(0.69,2.55)\end{array}$ & $\begin{array}{c}1.09 \\
(0.48,2.07)\end{array}$ & $\begin{array}{c}1.56 \\
(0.68,3.00)\end{array}$ & $\mathbf{I X A}$ & $\begin{array}{c}0.21 \\
(0.01,2.85)\end{array}$ & $\begin{array}{c}0.09 \\
(0.01,1.18)\end{array}$ & $\frac{\underline{0.12}}{(0.01,0.97)}$ & $\begin{array}{c}0.11 \\
(0.01,1.55)\end{array}$ & $\begin{array}{c}1.62 \\
(0.18,14.83)\end{array}$ & $\begin{array}{c}0.25 \\
(0.02,2.81)\end{array}$ & $\begin{array}{c}2.90 \\
(0.34,26.80)\end{array}$ & $\begin{array}{c}2.90 \\
(0.34,26.80)\end{array}$ \\
\hline $\begin{array}{c}0.91 \\
(0.65,1.24)\end{array}$ & $\begin{array}{c}\frac{1.27}{(1.05,1.52)} \\
\underline{1.5}\end{array}$ & $\begin{array}{c}1.35 \\
(0.83,2.3)\end{array}$ & $\begin{array}{c}1.32 \\
(0.93,1.9)\end{array}$ & $\begin{array}{c}0.99 \\
(0.79,1.22)\end{array}$ & $\begin{array}{c}\frac{1.42}{(1.12,1.77)} \\
\underline{1.12}\end{array}$ & $\begin{array}{c}0.99 \\
(0.54,1.77)\end{array}$ & ORT & $\begin{array}{c}0.42 \\
(0.06,3.48)\end{array}$ & $\begin{array}{c}0.53 \\
(0.13,2.50)\end{array}$ & $\begin{array}{c}0.51 \\
(0.07,4.51)\end{array}$ & $\begin{array}{c}7.47 \\
(0.59,104.75)\end{array}$ & $\begin{array}{c}1.14 \\
(0.12,9.43)\end{array}$ & $\begin{array}{c}112.89 \\
(1.07,211.14)\end{array}$ & $\begin{array}{c}112.89 \\
\underline{1.07,211.14}\end{array}$ \\
\hline $\begin{array}{c}\underline{0.72} \\
\underline{0.51,0.98)} \\
\end{array}$ & $\begin{array}{c}1.00 \\
(0.83,1.2)\end{array}$ & $\begin{array}{c}1.06 \\
(0.65,1.79)\end{array}$ & $\begin{array}{c}1.04 \\
(0.73,1.48)\end{array}$ & $\begin{array}{c}\underline{0.78} \\
\underline{0.63,0.96)} \\
\end{array}$ & $\begin{array}{c}1.12 \\
(0.88,1.39)\end{array}$ & $\begin{array}{c}0.78 \\
(0.43,1.38)\end{array}$ & $\begin{array}{c}\underline{0.79} \\
\underline{0.64 .0 .97)} \\
\end{array}$ & RAD-223 & $\begin{array}{c}1.27 \\
(0.29,5.64)\end{array}$ & $\begin{array}{c}1.20 \\
(0.14,10.48)\end{array}$ & $\begin{array}{c}\frac{18.12}{(1.30,266.12)} \\
(1.0)\end{array}$ & $\begin{array}{c}2.70 \\
(0.33,19.13)\end{array}$ & $\begin{array}{c}\underline{31.00} \\
\underline{(2.48,500.55)}\end{array}$ & $\begin{array}{c}\underline{31.00} \\
\underline{(2.48,500.55)} \\
\end{array}$ \\
\hline $\begin{array}{c}1.01 \\
(0.76,1.37)\end{array}$ & $\begin{array}{c}1.43 \\
\underline{(1.28,1.59)} \\
\end{array}$ & $\begin{array}{c}1.43 \\
(0.83,2.63)\end{array}$ & $\begin{array}{c}\underline{1.43} \\
\underline{(1.05,1.96)}\end{array}$ & $\begin{array}{c}1.11 \\
(0.93,1.32)\end{array}$ & $\stackrel{1.59}{(1.33,1.89)}$ & $\begin{array}{c}1.02 \\
(0.55,2.22)\end{array}$ & $\begin{array}{c}1.12 \\
(0.98,1.30)\end{array}$ & $\begin{array}{c}\underline{1.43} \\
(1.25,1.64)\end{array}$ & PRE & $\begin{array}{c}0.94 \\
(0.22,4.34)\end{array}$ & $\begin{array}{c}\underline{14.01} \\
\underline{(1.51,121.56}\end{array}$ & $\begin{array}{c}2.14 \\
(0.47,8.52)\end{array}$ & $\begin{array}{c}\underline{23.88} \\
\underline{(3.28,219.43)} \\
\end{array}$ & $\begin{array}{c}\underline{23.88} \\
\underline{(3.28,219.43)} \\
\end{array}$ \\
\hline $\begin{array}{c}1.01 \\
(0.7,1.39)\end{array}$ & $\begin{array}{c}1.40 \\
1.16,1.96 \\
\end{array}$ & $\begin{array}{c}1.49 \\
(0.91,2.55)\end{array}$ & $\begin{array}{c}1.47 \\
\underline{11.02 .2 .11} \\
\end{array}$ & $\begin{array}{c}1.10 \\
(0.86,1.36)\end{array}$ & $\frac{1.57}{(1.23,2.00)}$ & $\begin{array}{c}1.09 \\
(0.6,1.94)\end{array}$ & $\begin{array}{c}1.12 \\
(0.9,1.37)\end{array}$ & $\begin{array}{c}1.41 \\
(1.14,1.73) \\
\end{array}$ & $\begin{array}{c}0.98 \\
(0.83,1.14)\end{array}$ & SAT & $\begin{array}{c}14.30 \\
(1,00,192.52) \\
\end{array}$ & $\begin{array}{c}2.23 \\
(0.26,16.36)\end{array}$ & $\begin{array}{c}\frac{25.57}{(2.23,330.16} \\
.\end{array}$ & $\begin{array}{c}\underline{25.57} \\
(2.23,330.16 \\
\end{array}$ \\
\hline $\begin{array}{c}1.53 \\
(0.79,2.7)\end{array}$ & $\begin{array}{c}\underline{2.15} \\
\underline{(1.02 .4 .04)}\end{array}$ & $\begin{array}{c}2.25 \\
(0.99,4.5)\end{array}$ & $\begin{array}{c}2.16 \\
0.1,3.80 \\
\end{array}$ & $\begin{array}{c}1.68 \\
(0.79,3.18)\end{array}$ & $\begin{array}{c}\frac{2.4}{(1.13 .4 .59)} \\
\end{array}$ & $\begin{array}{c}1.65 \\
(0.69,3.34)\end{array}$ & $\begin{array}{c}1.71 \\
(0.81,3.23)\end{array}$ & $\begin{array}{c}\underline{2.16} \\
\underline{1.03,4.07)} \\
\underline{1.03}\end{array}$ & $\begin{array}{c}1.50 \\
(0.73,2.83)\end{array}$ & $\begin{array}{c}1.55 \\
(0.73,2.95)\end{array}$ & SIL & $\begin{array}{c}0.15 \\
(0.01,1.95)\end{array}$ & $\begin{array}{c}1.73 \\
(0.19,18.05)\end{array}$ & $\begin{array}{c}1.73 \\
(0.19,18.05)\end{array}$ \\
\hline $\begin{array}{c}0.94 \\
(0.66,1.3)\end{array}$ & $\begin{array}{c}\frac{1.31}{(1.05,1.62)} \\
\end{array}$ & $\begin{array}{c}1.39 \\
(0.84,2.42)\end{array}$ & $\begin{array}{c}1.37 \\
(0.94,1.99)\end{array}$ & $\begin{array}{c}1.03 \\
(0.8,1.29)\end{array}$ & $\begin{array}{c}1.47 \\
(1.13 .1 .89) \\
\end{array}$ & $\begin{array}{c}1.02 \\
(0.55,1.82)\end{array}$ & $\begin{array}{c}1.04 \\
(0.83,1.3)\end{array}$ & 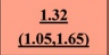 & $\begin{array}{c}0.92 \\
(0.77,1.10)\end{array}$ & $\begin{array}{c}0.94 \\
(0.74,1.19)\end{array}$ & $\begin{array}{c}0.70 \\
(0.38,1.28)\end{array}$ & SUN & $\begin{array}{c}11.23 \\
(1.07,170.1) \\
\end{array}$ & $\begin{array}{c}\underline{11.23} \\
\ldots 1.07,170.1) \\
\end{array}$ \\
\hline $\begin{array}{c}1.07 \\
(0.41,2.28)\end{array}$ & $\begin{array}{c}1.48 \\
(0.58,3.2)\end{array}$ & $\begin{array}{c}1.55 \\
(0.55,3.68)\end{array}$ & $\begin{array}{c}1.47 \\
(0.59,22.96)\end{array}$ & $\begin{array}{c}1.14 \\
(0.45,2.45)\end{array}$ & $\begin{array}{c}1.63 \\
(0.63,3.49)\end{array}$ & $\begin{array}{c}1.13 \\
(0.38,2.7)\end{array}$ & $\begin{array}{c}1.16 \\
(0.45,2.47)\end{array}$ & $\begin{array}{c}1.47 \\
(0.58,3.12)\end{array}$ & $\begin{array}{c}1.02 \\
(0.41,2.15)\end{array}$ & $\begin{array}{c}0.95 \\
(0.53,2.08)\end{array}$ & $\begin{array}{c}0.63 \\
(2.29,1.64)\end{array}$ & $\begin{array}{c}1.02 \\
(0.57,2.27)\end{array}$ & $\begin{array}{c}\text { RIL } \\
(15 \mathrm{mg} / \mathrm{kg})\end{array}$ & $\mathrm{NA}$ \\
\hline $\begin{array}{c}1.42 \\
(0.57,3.03)\end{array}$ & $\begin{array}{c}1.95 \\
(0.78,4.23)\end{array}$ & $\begin{array}{c}2.06 \\
(0.75,4.91)\end{array}$ & $\begin{array}{c}1.95 \\
(0.82,3.92)\end{array}$ & $\begin{array}{c}1.52 \\
(0.61,3.26)\end{array}$ & $\begin{array}{c}2.17 \\
(0.86,4.69)\end{array}$ & $\begin{array}{c}1.50 \\
(0.52,3.72)\end{array}$ & $\begin{array}{c}1.54 \\
(0.62,3.3)\end{array}$ & $\begin{array}{c}1.95 \\
(0.78,4.17)\end{array}$ & $\begin{array}{c}1.36 \\
(0.55,2.85)\end{array}$ & $\begin{array}{c}1.09 \\
(0.59,2.22)\end{array}$ & $\begin{array}{c}0.74 \\
(0.34,1.88)\end{array}$ & $\begin{array}{c}1.18 \\
(0.63,3.3)\end{array}$ & $\begin{array}{c}1.36 \\
(0.81,2.17)\end{array}$ & $\begin{array}{c}\text { RIL } \\
(7.5 \mathrm{mg} / \mathrm{kg})\end{array}$ \\
\hline
\end{tabular}

Figure 3. Pooled relative HRs for OS (pink region) and ORs for grade 3-4 adverse events (white region) based on mixed direct and indirect evidence from Bayesian network meta-analysis through fixed effects model with different pharmacological interventions in CRPC patients after docetaxel failure. The OS and safety estimates are located at the intersection of the column intervention and the row treatment (i.e., column intervention is reference for each comparison). To obtain HRs or ORs for comparisons in opposing direction, reciprocals should be applied. Results with statistic significant are in bold and underlined. Numbers in parentheses indicate $95 \%$ Crls for network meta-analysis.

\begin{tabular}{|c|c|c|c|c|c|c|c|c|c|c|c|c|}
\hline MIT & $\begin{array}{c}\underline{0.09} \\
(0.02,0.55) \\
\end{array}$ & $\begin{array}{c}2.50 \\
(0.55,13.92)\end{array}$ & $\begin{array}{c}2.45 \\
(0.54,10.73)\end{array}$ & $\begin{array}{c}0.15 \\
(0.02,1.24)\end{array}$ & $\begin{array}{c}\underline{0.06} \\
\underline{(0.01,0.49}\end{array}$ & $\begin{array}{c}0.14 \\
(0.02,1.16)\end{array}$ & $\begin{array}{c}\underline{0.08} \\
(0.02,0.34) \\
\end{array}$ & $\begin{array}{c}\underline{0.07} \\
\underline{(0.01,0.62)} \\
\end{array}$ & $\begin{array}{c}1.07 \\
(0.22,4.85)\end{array}$ & $\begin{array}{c}0.16 \\
(0.02,1.24)\end{array}$ & $\begin{array}{c}1.89 \\
(0.41,9.20)\end{array}$ & $\begin{array}{c}1.89 \\
(0.41,9.20)\end{array}$ \\
\hline $\begin{array}{c}0.97 \\
(0.74,1.24)\end{array}$ & $\mathbf{A A}$ & $\underline{\underline{(2.71,309.42)}}^{\frac{28.32}{3}}$ & $\begin{array}{c}\underline{26.97} \\
\underline{(2.79,284.16}\end{array}$ & $\begin{array}{c}1.65 \\
(0.29,9.08)\end{array}$ & $\begin{array}{c}0.70 \\
(0.12,3.71)\end{array}$ & $\begin{array}{c}1.61 \\
(0.27,8.52)\end{array}$ & $\begin{array}{c}0.86 \\
(0.35,2.03)\end{array}$ & $\begin{array}{c}0.80 \\
(0.16,5.25)\end{array}$ & $\frac{11.96}{\underline{(1.12,134.91)}}$ & $\begin{array}{c}1.85 \\
(0.30,9.36)\end{array}$ & 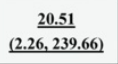 & $\frac{20.51}{(2.26,239.66)}$ \\
\hline $\begin{array}{c}1.19 \\
(0.72,1.83)\end{array}$ & $\begin{array}{c}1.23 \\
(0.69,2.05)\end{array}$ & CET & $\begin{array}{c}0.97 \\
(0.09,8.06)\end{array}$ & $\begin{array}{c}\underline{0.06} \\
(0.01,0.75) \\
\end{array}$ & $\begin{array}{c}\stackrel{0.02}{(0.01,0.29)} \\
\end{array}$ & $\begin{array}{c}\underline{0.06} \\
\underline{(0.01,0.69)}\end{array}$ & $\begin{array}{c}\underline{0.03} \\
(0.01,0.23) \\
\end{array}$ & $\begin{array}{c}\underline{0.03} \\
(0.01,0.36)\end{array}$ & $\begin{array}{c}0.42 \\
(0.04,3.84)\end{array}$ & $\begin{array}{c}\underline{0.07} \\
\underline{(0.01,0.71)}\end{array}$ & $\begin{array}{c}0.75 \\
(0.08,7.06)\end{array}$ & $\begin{array}{c}0.75 \\
(0.08,7.06)\end{array}$ \\
\hline$\stackrel{\frac{0.74}{(0.64,0.86)}}{\underline{0}}$ & $\begin{array}{c}0.76 \\
(0.55,1.04)\end{array}$ & $\begin{array}{c}\stackrel{0.63}{(0.41,0.93)} \\
\end{array}$ & CBA & $\begin{array}{c}\underline{0.06} \\
(0.01,0.88) \\
\end{array}$ & $\begin{array}{c}\underline{0.03} \\
(0.01,0.33) \\
\end{array}$ & $\begin{array}{c}\underline{0.06} \\
\underline{(0.01,0.79)}\end{array}$ & $\begin{array}{c}\underline{0.03} \\
(0.01,0.27) \\
\end{array}$ & $\begin{array}{c}\underline{0.03} \\
(0.01,0.44)\end{array}$ & $\begin{array}{c}0.43 \\
(0.05,4.00)\end{array}$ & $\begin{array}{c}\underline{0.07} \\
(0.01,0.96) \\
\end{array}$ & $\begin{array}{c}0.76 \\
(0.10,7.68)\end{array}$ & $\begin{array}{c}0.76 \\
(0.10,7.68)\end{array}$ \\
\hline$\underline{\underline{0.63}} \underline{\underline{(0.47,0.83)}}$ & $\underline{\underline{0.65}}$ & $\begin{array}{c}\underline{0.53} \\
\underline{(0.33,0.82)}\end{array}$ & $\begin{array}{c}0.85 \\
(0.60,1.18)\end{array}$ & CBO & $\begin{array}{c}0.42 \\
(0.05,3.25)\end{array}$ & $\begin{array}{c}0.98 \\
(0.13,7.30)\end{array}$ & $\begin{array}{c}0.53 \\
(0.12,2.24)\end{array}$ & $\begin{array}{c}0.49 \\
(0.06,4.40)\end{array}$ & $\begin{array}{c}7.39 \\
(0.52,99.21)\end{array}$ & $\begin{array}{c}1.11 \\
(0.14,8.42)\end{array}$ & $\begin{array}{c}\underline{12.49} \\
\underline{(1.06,192.70)}\end{array}$ & $\begin{array}{c}\frac{12.49}{(1.06,192.70)} \\
\underline{10}\end{array}$ \\
\hline $\begin{array}{c}\underline{0.52} \\
(0.40,0.68)\end{array}$ & $\begin{array}{c}\underline{0.54} \\
\underline{(0.45,0.65)}\end{array}$ & $\begin{array}{c}\stackrel{0.45}{(0.28,0.68)} \\
\end{array}$ & $\begin{array}{c}\stackrel{0.71}{(0.50,0.97)} \\
\end{array}$ & $\begin{array}{c}0.83 \\
(0.67,1.03)\end{array}$ & ENZ & $\begin{array}{c}2.30 \\
(0.27,19.01)\end{array}$ & $\begin{array}{c}1.22 \\
(0.31,5.60)\end{array}$ & $\begin{array}{c}1.17 \\
(0.16,11.23)\end{array}$ & $\begin{array}{c}\frac{17.15}{(1.24,261.44)} \\
\end{array}$ & $\begin{array}{c}2.64 \\
(0.33,20.47)\end{array}$ & $\begin{array}{c}\underline{29.43} \\
\underline{(2.51,478.27)} \\
\end{array}$ & $\begin{array}{c}\stackrel{29.43}{(2.51,478.27)} \\
\end{array}$ \\
\hline $\begin{array}{c}1.00 \\
(0.76,1.29)\end{array}$ & $\begin{array}{c}1.03 \\
(0.84,1.24)\end{array}$ & $\begin{array}{c}0.85 \\
(0.53,1.29)\end{array}$ & $\begin{array}{c}1.35 \\
(0.95,1.86)\end{array}$ & $\begin{array}{c}\frac{1.59}{(1.26,1.98)} \\
\end{array}$ & $\frac{1.91}{(1.54,2.35)}$ & ORT & $\begin{array}{c}0.53 \\
(0.13,2.50)\end{array}$ & $\begin{array}{c}0.51 \\
(0.07,4.51)\end{array}$ & $\begin{array}{c}7.47 \\
(0.59,104.75)\end{array}$ & $\begin{array}{c}1.14 \\
(0.12,9.43)\end{array}$ & $\begin{array}{c}\underline{12.89} \\
\underline{(1.07,211.14)}\end{array}$ & $\begin{array}{c}\underline{12.89} \\
\underline{(1.07,211.14}\end{array}$ \\
\hline $\begin{array}{c}\frac{1.33}{(1.02,1.69)} \\
\end{array}$ & $\begin{array}{c}\frac{1.35}{(1.20,1.53)} \\
\end{array}$ & $\begin{array}{c}1.10 \\
(0.66,1.89)\end{array}$ & $\stackrel{1.75}{(1.33,2.33)}$ & $\begin{array}{c}\frac{2.08}{(1.75,2.50)} \\
\end{array}$ & $\begin{array}{c}\underline{2.5} \\
\underline{(2.17,2.86)} \\
\end{array}$ & $\frac{1.32}{(1.12,1.54)}$ & PRE & $\begin{array}{c}0.94 \\
(0.22,4.34)\end{array}$ & $\begin{array}{c}\underline{14.01} \\
\underline{(1.51,121.56} \\
\end{array}$ & $\begin{array}{c}2.14 \\
(0.47,8.52)\end{array}$ & $\begin{array}{c}\underline{23.88} \\
\underline{(3.28,219.43)} \\
\end{array}$ & $\begin{array}{c}\underline{23.88} \\
\underline{(3.28,219.43)} \\
\end{array}$ \\
\hline $\begin{array}{c}1.5 \\
(0.83,2.56)\end{array}$ & $\begin{array}{c}1.18 \\
(0.63,2.05)\end{array}$ & $\begin{array}{c}0.97 \\
(0.48,176)\end{array}$ & $\begin{array}{c}1.55 \\
(0.88,2.59)\end{array}$ & $\begin{array}{c}1.82 \\
(0.97,3.16)\end{array}$ & $\stackrel{\frac{2.19}{(1.16,3.82)}}{\underline{1}}$ & $\begin{array}{c}1.15 \\
(0.61,2.00)\end{array}$ & $\begin{array}{c}1.00 \\
(0.85,1.16)\end{array}$ & SAT & $\begin{array}{l}\frac{14.30}{(1.00,192.52)} \\
\underline{1.0}\end{array}$ & $\begin{array}{c}2.23 \\
(0.26,16.36)\end{array}$ & $\begin{array}{l}\frac{25.57}{(2.23,330.16} \\
\end{array}$ & $\begin{array}{l}\frac{25.57}{(2.23,330.16)} \\
\end{array}$ \\
\hline $\begin{array}{c}1.21 \\
(0.66,2.09)\end{array}$ & $\begin{array}{c}0.95 \\
(0.50,1.66)\end{array}$ & $\begin{array}{c}0.78 \\
(0.38,1.43)\end{array}$ & $\begin{array}{c}1.25 \\
(0.70,2.10)\end{array}$ & $\begin{array}{c}1.47 \\
(0.76,2.55)\end{array}$ & $\begin{array}{c}1.77 \\
(0.92,3.14)\end{array}$ & $\begin{array}{c}0.93 \\
(0.48,1.63)\end{array}$ & $\begin{array}{c}1.36 \\
(0.71,2.35)\end{array}$ & $\begin{array}{c}1.44 \\
(0.85,2.36)\end{array}$ & SIL & $\begin{array}{c}0.15 \\
(0.01,1.95)\end{array}$ & $\begin{array}{c}1.73 \\
(0.19,18.05)\end{array}$ & $\begin{array}{c}1.73 \\
(0.19,18.05)\end{array}$ \\
\hline $\begin{array}{c}1.31 \\
(0.98,1.7)\end{array}$ & $\begin{array}{c}\frac{1.35}{(1.11,1.65} \\
\end{array}$ & $\begin{array}{c}1.11 \\
(0.70,1.71)\end{array}$ & $\begin{array}{c}\frac{1.78}{(1.26,2.45} \\
\end{array}$ & $\begin{array}{c}\underline{2.08} \\
\underline{(1.65,2.60)} \\
\end{array}$ & $\begin{array}{c}\underline{2.5} \\
\underline{(2.01,3.07)} \\
\end{array}$ & $\begin{array}{c}\frac{1.32}{(1.06,1.62)} \\
\end{array}$ & $\begin{array}{c}\underline{0.73} \\
\underline{(0.60,0.89)} \\
\end{array}$ & $\begin{array}{c}0.73 \\
(0.56,0.92)\end{array}$ & $\begin{array}{c}0.54 \\
(0.30,0.94)\end{array}$ & SUN & $\begin{array}{c}\underline{11.23} \\
(1.07,170.16) \\
\end{array}$ & $\begin{array}{c}\underline{11.23} \\
(1.07,170.16 \\
\end{array}$ \\
\hline $\begin{array}{c}\underline{1.78} \\
(1.00,2.94) \\
\end{array}$ & $\begin{array}{c}1.84 \\
(0.95,3.22)\end{array}$ & $\begin{array}{c}1.51 \\
(0.73,2.81)\end{array}$ & $\begin{array}{c}\frac{2.41}{(1.32,4.04)} \\
(.5)\end{array}$ & $\begin{array}{c}\underline{2.83} \\
\underline{(1.45,4.99)}\end{array}$ & $\begin{array}{c}\underline{3.41} \\
\underline{(1.74,6.00)}\end{array}$ & $\begin{array}{c}\frac{1.79}{(0.91,3.12)} \\
\end{array}$ & $\begin{array}{c}0.88 \\
(0.48,1.50)\end{array}$ & $\begin{array}{c}0.91 \\
(0.55,1.69)\end{array}$ & $\begin{array}{c}0.66 \\
(0.35,1.47)\end{array}$ & $\begin{array}{c}1.25 \\
(0.77,2.33)\end{array}$ & $\begin{array}{c}\text { RIL } \\
(15 \mathrm{mg} / \mathrm{kg})\end{array}$ & $\mathrm{NA}$ \\
\hline $\begin{array}{c}0.95 \\
(0.70,1.27)\end{array}$ & $\begin{array}{c}0.98 \\
(0.78,1.24)\end{array}$ & $\begin{array}{c}0.81 \\
(0.50,1.27)\end{array}$ & $\begin{array}{c}1.29 \\
(0.90,1.80)\end{array}$ & $\frac{1.51}{(1.17,1.95)}$ & $\begin{array}{c}\frac{1.82}{(1.42,2.31)} \\
\end{array}$ & $\begin{array}{c}0.96 \\
(0.75,1.22)\end{array}$ & $\begin{array}{c}0.71 \\
(0.37,1.21)\end{array}$ & $\begin{array}{c}0.69 \\
(0.53,1.18)\end{array}$ & $\begin{array}{c}0.51 \\
(0.27,1.11)\end{array}$ & $\begin{array}{c}0.95 \\
(0.58,1.67)\end{array}$ & $\begin{array}{c}0.80 \\
(0.52,1.19)\end{array}$ & $\begin{array}{c}\text { RIL } \\
(7.5 \mathrm{mg} / \mathrm{kg})\end{array}$ \\
\hline
\end{tabular}

Treatment

Progression free survival $(\mathrm{HR}, 95 \% \mathrm{Crl})$

Adverse events $(\mathrm{OR}, 95 \% \mathrm{Crl})$

Figure 4. Pooled relative HRs for PFS (green region) and ORs for grade 3-4 adverse events (white region) based on mixed direct and indirect evidence from Bayesian network meta-analysis through fixed effects model with different pharmacological interventions in CRPC patients after docetaxel failure. The PFS and safety estimates are located at the intersection of the column intervention and the row treatment (i.e., column intervention is reference for each comparison). To obtain HRs or ORs for comparisons in opposing direction, reciprocals should be applied. Results with statistic significant are in bold and underlined. Numbers in parentheses indicate $95 \%$ Crls for network meta-analysis. 


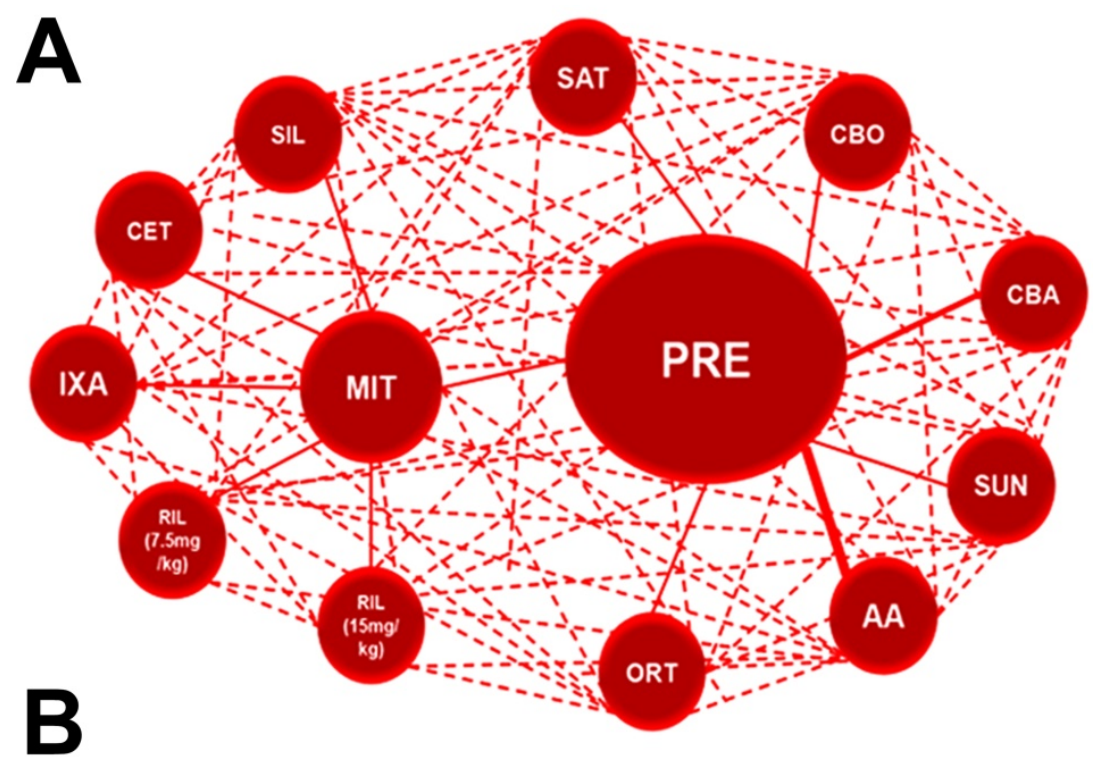

\begin{tabular}{|cccc|}
\hline Comparison & HR & 95\%CrI-Low & 95\%CrI-High \\
\hline AA vs PRE & 0.7016 & 0.6274 & 0.7839 \\
\hline CBA vs PRE & 0.6955 & 0.5010 & 0.9244 \\
\hline CET vs PRE & 0.7003 & 0.3682 & 1.2140 \\
\hline ORT vs PRE & 0.8893 & 0.7662 & 1.0260 \\
\hline CBO vs PRE & 0.9035 & 0.7621 & 1.0620 \\
\hline SUN vs PRE & 0.9165 & 0.7634 & 1.0900 \\
\hline LXA vs PRE & 0.9676 & 0.4489 & 1.8670 \\
\hline MIT vs PRE & 0.9782 & 0.7193 & 1.2770 \\
\hline SAT vs PRE & 0.9848 & 0.8359 & 1.1490 \\
\hline RIL(7.5mg/kg) vs PRE & 1.0140 & 0.3682 & 2.1450 \\
\hline RIL(15mg/kg) vs PRE & 1.3490 & 0.4925 & 2.9510 \\
\hline SIL vs PRE & 1.4860 & 0.7180 & 2.7390 \\
\hline
\end{tabular}

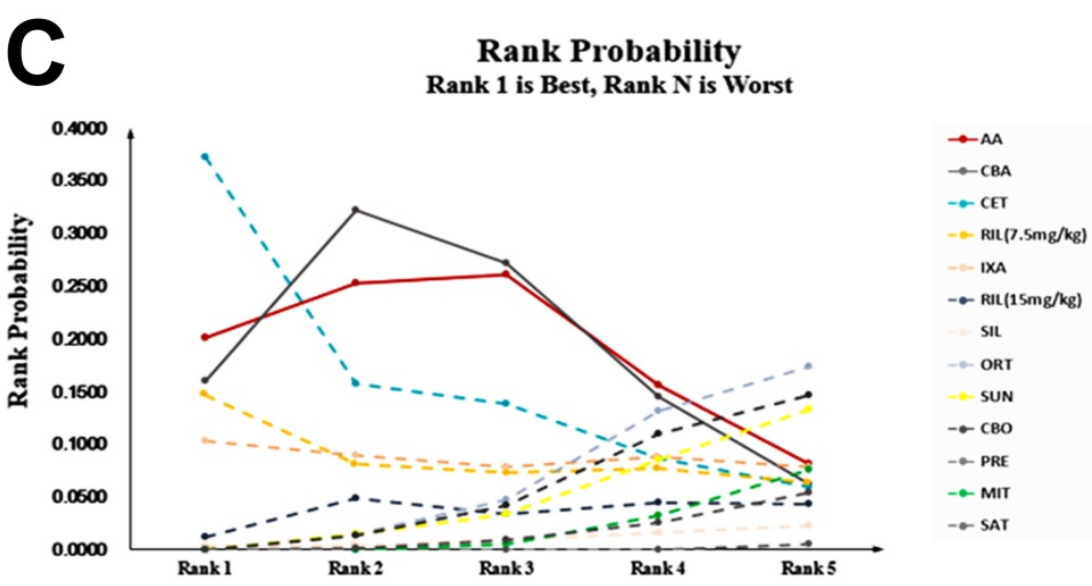

Figure 5. The network meta-analysis outcomes of eligible comparisons of OS excluding studies with control arm of placebo: network diagram (A), relative HRs (B) and rank probability $(\mathrm{C})$ based on mixed direct and indirect evidence from Bayesian network meta-analysis through fixed effects model with different pharmacological interventions in CRPC patients after docetaxel failure.

\section{Sensitivity analysis}

Theoretically daily low dose corticosteroids appear to have modest antitumor activity and may avert adverse effects of other antitumor agents ${ }^{12}$, we performed further pooled analysis including studies with control arm of Prednisone plus placebo. Our results showed that Abiraterone Acetate (HR: 0.71, 95\%CrI: 0.63-0.78) was the most efficacious intervention of being rank 1 in OS compared with Prednisone plus placebo followed by Cabazitaxel and Cetuximab, when Enzalutamide and Radium-223 were excluded for control arm of placebo (Figure 5). 
In terms of PFS, Abiraterone Acetate (HR: 0.74, 95\% CrI: 0.66-0.83) also presented the most efficacious intervention of being rank 1 (Supplementary Figure 3). In regards to PSA response, Abiraterone Acetate showed significant superiority over control arm (OR: 6.17, 95\%CrI: 2.83-13.59) (Supplementary Figure 4). Together, our results suggest that Abiraterone Acetate might be the efficacious intervention compared with control arm in terms of OS, PFS and PSA Response.

\section{Risk of bias assessment}

The risk of bias of included studies was reported in Figure 6. In fact, none of the trials were thought to have a high risk of bias for any of the methodological quality items assessed. In summary, 13 (76\%) of the 17 trials reported an adequate method of allocation concealment and 14 (82\%) of the 17 trials reported low risk method for blinding of outcomes. We did not find evidence of publication bias in OS, PSA response and adverse event, based on funnel plot asymmetry or quantitatively (Egger's regression test $p=0.266,0.178$ and 0.084), while Egger's regression test in PFS showed statistical significance $(\mathrm{p}=0.038)$ (Supplementary Figure 5).

\section{Discussion}

This systematic review and network meta-analysis represents the most comprehensive synthesis of data for currently available pharmacological interventions for patients with mCRPC who had progressed after docetaxel-based chemotherapy. 17 RCTs with 12347 enrolled patients were included in this network meta-analysis. The main new finding is that Abiraterone Acetate, Cabazitaxel and Enzalutamide presented better benefits in term of OS compared with control arms. Enzalutamide demonstrated superiority over PFS and PSA response with a highest probability to rank 1 . Moreover, sensitivity analysis showed that Abiraterone Acetate exhibited the most efficacious intervention of being rank 1 in term of OS compared with control, followed by Cabazitaxel and Cetuximab. In regards to PFS, Abiraterone Acetate also presented the most efficacious intervention of being rank 1 . Together, our results suggested that Abiraterone Acetate might be the efficacious intervention compared with control arms for mCRPC patients progressing after docetaxel-based chemotherapy.

Previously, post-docetaxel treatment options for mCRPC were limited, with few benefits observed in terms of OS. Since 2010, we have witnessed unprecedented therapeutic advances in treatment after prior docetaxel-based chemotherapy for men with mCRPC, including Cabazitaxel (tubulin-binding taxane) $)^{15} 28$ approved by the FDA in 2010, Abiraterone
Acetate (androgen biosynthesis inhibitor) ${ }^{12} \quad 46 \quad 47$ approved by the FDA in 2011 and Enzalutamide (androgen receptor antagonist) ${ }^{13} 38$ 48-50 approved by the FDA in 2012. All of the regimens have been demonstrated to improve efficacy and have become parts of the therapeutic arsenals mCRPC after docetaxel failure. An important finding within the present study is that, Abiraterone Acetate appears to be efficacious treatment option for mCRPC patients after docetaxel failure because of the higher efficacy and lower adverse events. Abiraterone Acetate, a steroidal drug, inhibits CYP17A1, blocks androgen synthesis decreasing the intracellular testosterone level and prolong survival before or after docetaxel chemotherapy, recent study has indicated the metabolites of Abiraterone Acetate showed antagonistic effect on androgen receptor 124751 . It might partially explain the outcomes in our study.

Entering this new therapeutic era in oncology, numbers of mechanistic drug classifications have produced a more diverse range of potential toxicities. CTCAE is a standard evaluating system designed to assess symptomatic toxicities and provide additional tolerability data ${ }^{52}$. The interventions included in our study composed of chemotherapy, hormonal strategies, antiangiogenic therapies, immune strategies and bone-targeting agents, and the assessments of severe adverse events (Grade 3-4) could better inform us the tolerability information of the therapies. Our results indicated reported adverse events of hormone strategies were less severe than cytotoxic agents, and were manageable by appropriate patient monitoring. The novel androgen receptor targeting agents (Abiraterone Acetate and Enzalutamide) presented similar severe adverse events with control arms, which could be better tolerated than cytotoxic therapies.

To the best of our knowledge, this study is the first network meta-analysis to assess the efficacy and safety of different interventions for post-docetaxel therapy in mCRPC patients. We overcame the difficulties of different measures of survival across studies and synthesized all available studies within a single network meta-analysis, avoiding potential selection bias in the meantime. This network meta-analysis provides new insights into controversies on this issue with important implications in clinical care and future research. However, this study also has limitation. We extracted all information from published data rather than original individual patient data, which may lead to publication and reporting bias and missing information on certain endpoints might affected our analysis without access to individual patient data. We minimized the risk of bias through searching and 
reviewing the publications comprehensively, and extracting and evaluating the data systematically, we also performed further pooled analysis excluding agents with control arm of placebo for theoretical reasons. Moreover, our findings are the results of direct and indirect comparisons in a network meta-analysis. Although this method is widely accepted, it does not substitute results from RCTs. Future systematic reviews are needed to assess the efficacy and safety of these interventions by identifying those patients who most benefit.

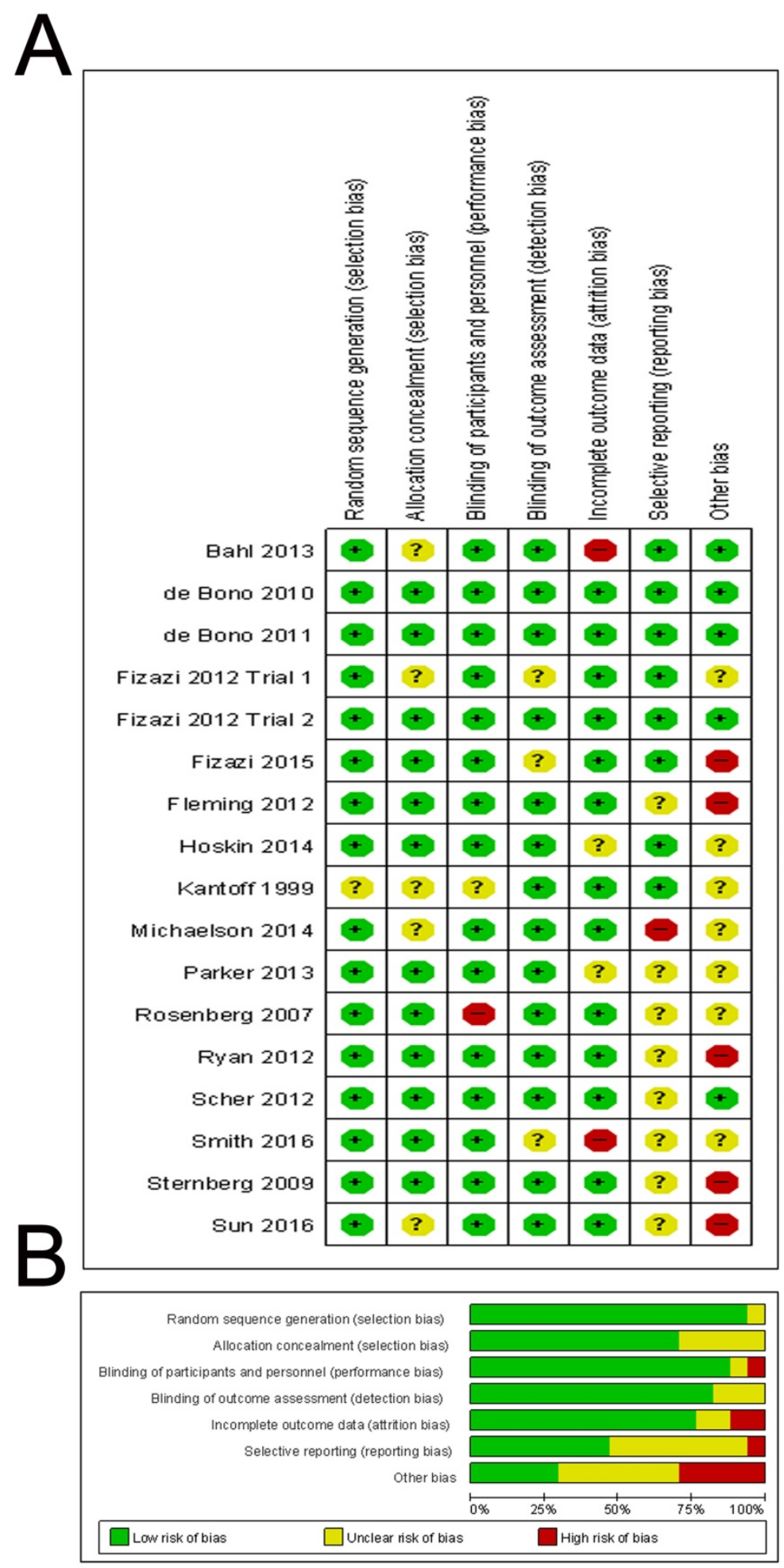

Figure 6. Quality assessment of included studies. The Overall (A) and Study-level distribution plot for risk of bias using Cochrane's risk of bias assessment tool. Studies are deemed to be at high, low or unclear risk of bias for each risk of bias entry. The review authors' judgments about each risk of bias entry are presented as percentages across all included studies. 


\section{Conclusions}

In conclusion, our network meta-analysis demonstrated that Abiraterone Acetate, Cabazitaxel and Enzalutamide were associated with favorable OS when compared with control arms. Abiraterone Acetate appears to be efficacious treatment option for mCRPC patients after docetaxel failure because of the higher efficacy and lower adverse events. Similar observations were also noted for PFS and PSA response. Further sensitivity analysis indicated Abiraterone Acetate showed significant benefit in prolonging survival. Future well-designed RCTs and systematic reviews are awaited to confirm the findings of this study.

\section{Clinical Practice Points}

- The consensus is not existing of optimal strategies for CRPC patients after docetaxel failure. Several randomized trials were conducted to investigate efficacy and safety of interventions.

- This network meta-analysis showed for the first time that Abiraterone Acetate (HR: 0.71, 95\% CrI: 0.63-0.78) exhibited the most efficacious intervention of being rank 1 in term of OS compared with control arms.

- The analysis of 17 randomized trials provides evidence in favor of Abiraterone Acetate for CRPC patients after docetaxel failure with acceptable tolerability and good performance status.

\section{Abbreviations}

PCa: Prostate cancer; ADT: Androgen deprivation therapy; CRPC: Castration Resistant Prostate Cancer; OS: Overall Survival; PFS: Progression Fee Survival; RCT: Randomized Controlled Trials; PRISMA: Preferred Reporting Items for Systematic Reviews and Meta-analyses; HR: Hazard Ratio; OR: Odds Ratio; CrIs: Credible Intervals; DIC: Deviance Information Criterion; CTCAE: Common Terminology Criteria for Adverse Events; EAU: European Association of Urology; NCCN: National Comprehensive Cancer Network; MIT: Mitoxantrone; CBA: Cabazitaxel; PRE: Prednisone plus Placebo; AA: Abiraterone acetate; SIL: Siltuximab; ORT: Orteronel; CET: Cetuximab; RAD: Radium-223; SUN: Sunitinib; IXA: Ixabepilone; RIL: Rilotumumab; ENZ: Enzalutamide; CBO: Cabozantinib; SAT: Satraplatin.

\section{Supplementary Material}

Supplementary figures and tables. http://www.jcancer.org/v09p0690s1.pdf

\section{Acknowledgements}

This study was funded by the National Natural Science Foundation of China (Grant No. 81572514, U1301221, 81472384, 81402106, 81372729, 81272808, 81172431), National Natural Science Foundation of Guangdong (Grant No. 2016A030313321, 2015A030 311011，2015A030310122, S2013020012671), Science and Technology Program of Guangzhou (Grant No. 201604020156, 201604020177), "Three Big Constructions" funds of Sun Yat-sen University (for Jian Huang and Tianxin Lin), Specialized Research Fund for the Doctoral Program of Higher Education (for Tianxin Lin, 20130171110073), the Fundamental Research Funds for the Central Universities (for Jian Huang), Project Supported by Guangdong Province Higher Vocational Colleges \& Schools Pearl River Scholar Funded Scheme (for Tianxin Lin), Elite Young Scholars Program of Sun Yat-Sen Memorial Hospital (for Tianxin Lin, J201401) and National Clinical Key Specialty Construcion Project for Department of Urology and Department of Oncology. Grant KLB09001 from the Key Laboratory of Malignant Tumor Gene Regulation and Target Therapy of Guangdong Higher Education Institutes, Sun-Yat-Sen University. Grant [2013]163 from Key Laboratory of Malignant Tumor Molecular Mechanism and Translational Medicine of Guangzhou Bureau of Science and Information Technology.

\section{Competing Interests}

The authors have declared that no competing interest exists.

\section{References}

1. Ferlay J, Soerjomataram I, Dikshit R, et al. Cancer incidence and mortality worldwide: sources, methods and major patterns in GLOBOCAN 2012. Int J Cancer 2015;136(5):E359-86. doi: 10.1002/ijc.29210 [published Online First: 2014/09/16]

2. Siegel RL, Miller KD, Jemal A. Cancer Statistics, 2017. CA: a cancer journal for clinicians 2017;67(1):7-30. doi: 10.3322/caac.21387

3. Beltran H, Beer TM, Carducci MA, et al. New therapies for castration-resistant prostate cancer: efficacy and safety. European urology 2011;60(2):279-90. doi: 10.1016/j.eururo.2011.04.038

4. Heidenreich A, Bastian PJ, Bellmunt J, et al. EAU guidelines on prostate cancer. Part II: Treatment of advanced, relapsing, and castration-resistant prostate cancer. European urology 2014;65(2):467-79. doi: 10.1016/j.eururo.2013.11.002

5. Loblaw DA, Virgo KS, Nam $\mathrm{R}$, et al. Initial hormonal management of androgen-sensitive metastatic, recurrent, or progressive prostate cancer: 2006 update of an American Society of Clinical Oncology practice guideline. Journal of clinical oncology : official journal of the American Society of Clinical Oncology 2007:25(12):1596-605. doi: 10.1200/JCO.2006.10.1949

6. Pagliarulo V, Bracarda S, Eisenberger MA, et al. Contemporary role of androgen deprivation therapy for prostate cancer. European urology 2012:61(1):11-25 doi: 10.1016/j.eururo.2011.08.026

7. Chowdhury S, Burbridge S, Harper PG. Chemotherapy for the treatment of hormone-refractory prostate cancer. International journal of clinical practice 2007;61(12):2064-70. doi: 10.1111/j.1742-1241.2007.01551.x 
8. Parker C, Gillessen S, Heidenreich A, et al. Cancer of the prostate: ESMO Clinical Practice Guidelines for diagnosis, treatment and follow-up. Annals of oncology : official journal of the European Society for Medical Oncology 2015;26 Suppl 5:v69-77. doi: 10.1093/annonc/mdv222

9. Lam JS, Leppert JT, Vemulapalli SN, et al. Secondary hormonal therapy for advanced prostate cancer. The Journal of urology 2006;175(1):27-34. doi: 10.1016/S0022-5347(05)00034-0

10. Mohler JL, Armstrong AJ, Bahnson RR, et al. Prostate Cancer, Version 1.2016. J Natl Compr Canc Netw 2016;14(1):19-30.

11. Berthold DR, Pond GR, Soban F, et al. Docetaxel plus prednisone or mitoxantrone plus prednisone for advanced prostate cancer: updated survival in the TAX 327 study. Journal of clinical oncology : official journal of the American Society of Clinical Oncology 2008;26(2):242-5. doi: 10.1200/JCO.2007.12.4008

12. de Bono JS, Logothetis CJ, Molina A, et al. Abiraterone and increased survival in metastatic prostate cancer. The New England journal of medicine 2011;364(21):1995-2005. doi: 10.1056/NEJMoa1014618

13. Scher HI, Fizazi K, Saad F, et al. Increased survival with enzalutamide in prostate cancer after chemotherapy. N Engl J Med 2012;367(13):1187-97. doi: 10.1056/NEJMoa1207506

14. Parker C, Nilsson S, Heinrich D, et al. Alpha emitter radium-223 and survival in metastatic prostate cancer. N Engl J Med 2013;369(3):213-23. doi: 10.1056/NEJMoa1213755

15. de Bono JS, Oudard S, Ozguroglu M, et al. Prednisone plus cabazitaxel or mitoxantrone for metastatic castration-resistant prostate cancer progressing after docetaxel treatment: a randomised open-label trial. Lancet 2010;376(9747):1147-54. doi: 10.1016/S0140-6736(10)61389-X

16. Loriot $\mathrm{Y}$, Bianchini D, Ileana E, et al. Antitumour activity of abiraterone acetate against metastatic castration-resistant prostate cancer progressing after docetaxel and enzalutamide (MDV3100). Annals of oncology : official journal of the European Society for Medical Oncology 2013;24(7):1807-12. doi: $10.1093 /$ annonc/mdt136

17. Noonan KL, North S, Bitting RL, et al. Clinical activity of abiraterone acetate in patients with metastatic castration-resistant prostate cancer progressing after enzalutamide. Annals of oncology : official journal of the European Society for Medical Oncology 2013;24(7):1802-7. doi: 10.1093/annonc/mdt138

18. Bianchini D, Lorente D, Rodriguez-Vida A, et al. Antitumour activity of enzalutamide (MDV3100) in patients with metastatic castration-resistant prostate cancer (CRPC) pre-treated with docetaxel and abiraterone. Eur I Cancer 2014;50(1):78-84. doi: 10.1016/j.ejca.2013.08.020

19. Cipriani A, Higgins JP, Geddes JR, et al. Conceptual and technical challenges in network meta-analysis. Annals of internal medicine 2013;159(2):130-7. doi: 10.7326/0003-4819-159-2-201307160-00008

20. Lu G, Ades AE. Combination of direct and indirect evidence in mixed treatment comparisons. Statistics in medicine 2004;23(20):3105-24. doi: $10.1002 / \operatorname{sim} .1875$

21. Caldwell DM, Ades AE, Higgins JP. Simultaneous comparison of multiple treatments: combining direct and indirect evidence. Bmj 2005;331(7521):897-900. doi: 10.1136/bmj.331.7521.897

22. Liberati A, Altman DG, Tetzlaff J, et al. The PRISMA statement for reporting systematic reviews and meta-analyses of studies that evaluate health care interventions: explanation and elaboration. Journal of clinical epidemiology 2009;62(10):e1-34. doi: 10.1016/j.jclinepi.2009.06.006

23. Moher D, Liberati A, Tetzlaff J, et al. Preferred reporting items for systematic reviews and meta-analyses: the PRISMA statement. Journal of clinical epidemiology 2009;62(10):1006-12. doi: 10.1016/j.jclinepi.2009.06.005

24. Tannock IF, Osoba D, Stockler MR, et al. Chemotherapy with mitoxantrone plus prednisone or prednisone alone for symptomatic hormone-resistant prostate cancer: a Canadian randomized trial with palliative end points. J Clin Oncol 1996;14(6):1756-64. doi: 10.1200/JCO.1996.14.6.1756

25. Tannock IF, de Wit R, Berry WR, et al. Docetaxel plus prednisone or mitoxantrone plus prednisone for advanced prostate cancer. $N$ Engl J Med 2004:351(15):1502-12. doi: 10.1056/NEJMoa040720

26. Morgan CJ, Oh WK, Naik G, et al. Impact of prednisone on toxicities and survival in metastatic castration-resistant prostate cancer: A systematic review and meta-analysis of randomized clinical trials. Crit Rev Oncol Hematol 2014;90(3):253-61. doi: 10.1016/j.critrevonc.2013.12.001

27. Sonpavde G, Pond GR, Templeton AJ, et al. Impact of single-agent daily prednisone on outcomes in men with metastatic castration-resistant prostate cancer. Prostate Cancer Prostatic Dis 2017;20(1):67-71. doi: 10.1038/pcan.2016.44

28. Bahl A, Oudard S, Tombal B, et al. Impact of cabazitaxel on 2-year survival and palliation of tumour-related pain in men with metastatic castration-resistant prostate cancer treated in the TROPIC trial. Ann Oncol 2013;24(9):2402-8. doi: 10.1093/annonc/mdt194

29. Higgins JP, Altman DG, Gotzsche PC, et al. The Cochrane Collaboration's tool for assessing risk of bias in randomised trials. Bmj 2011;343:d5928. doi: 10.1136/bmj.d5928

30. Salanti G, Ades AE, Ioannidis JP. Graphical methods and numerical summaries for presenting results from multiple-treatment meta-analysis: an overview and tutorial. Journal of clinical epidemiology 2011;64(2):163-71. doi: 10.1016/j.jclinepi.2010.03.016

31. Parmar MK, Torri V, Stewart L. Extracting summary statistics to perform meta-analyses of the published literature for survival endpoints. Statistics in medicine 1998;17(24):2815-34.

32. Fleming MT, Sonpavde G, Kolodziej M, et al. Association of rash with outcomes in a randomized phase II trial evaluating cetuximab in combination with mitoxantrone plus prednisone after docetaxel for metastatic castration-resistant prostate cancer. Clin Genitourin Cancer 2012;10(1):6-14. doi: 10.1016/j.clgc.2011.11.003

33. Kantoff PW, Halabi S, Conaway M, et al. Hydrocortisone with or without mitoxantrone in men with hormone-refractory prostate cancer: results of the cancer and leukemia group B 9182 study. J Clin Oncol 1999;17(8):2506-13. doi: 10.1200/JCO.1999.17.8.2506

34. Rosenberg JE, Weinberg VK, Kelly WK, et al. Activity of second-line chemotherapy in docetaxel-refractory hormone-refractory prostate cancer patients : randomized phase 2 study of ixabepilone or mitoxantrone and prednisone. Cancer 2007;110(3):556-63. doi: 10.1002/cncr.22811

35. Ryan CJ, Rosenthal $\mathrm{M}, \mathrm{Ng} \mathrm{S}$, et al. Targeted MET inhibition in castration-resistant prostate cancer: a randomized phase II study and biomarker analysis with rilotumumab plus mitoxantrone and prednisone. Clin Cancer Res 2013;19(1):215-24. doi: 10.1158/1078-0432.CCR-12-2605

36. Egger M, Davey Smith $G$, Schneider $M$, et al. Bias in meta-analysis detected by a simple, graphical test. Bmj 1997;315(7109):629-34

37. Cella D, Ivanescu C, Holmstrom S, et al. Impact of enzalutamide on quality of life in men with metastatic castration-resistant prostate cancer after chemotherapy: additional analyses from the AFFIRM randomized clinical trial. Annals of oncology : official journal of the European Society for Medical Oncology 2015;26(1):179-85. doi: 10.1093/annonc/mdu510

38. Fizazi K, Scher HI, Miller K, et al. Effect of enzalutamide on time to first skeletal-related event, pain, and quality of life in men with castration-resistant prostate cancer: results from the randomised, phase 3 AFFIRM trial. Lancet Oncol 2014;15(10):1147-56. doi: 10.1016/S1470-2045(14)70303-1

39. Logothetis CJ, Basch E, Molina A, et al. Effect of abiraterone acetate and prednisone compared with placebo and prednisone on pain control and skeletal-related events in patients with metastatic castration-resistant prostate cancer: exploratory analysis of data from the COU-AA-301 randomised trial. Lancet Oncol 2012;13(12):1210-7. doi: 10.1016/S1470-2045(12)70473-4

40. Sartor O, Coleman R, Nilsson S, et al. Effect of radium-223 dichloride on symptomatic skeletal events in patients with castration-resistant prostate cancer and bone metastases: results from a phase 3, double-blind, randomised trial. Lancet Oncol 2014;15(7):738-46. doi: 10.1016/S1470-2045(14)70183-4

41. Saad F, Hotte S, North S, et al. Randomized phase II trial of Custirsen (OGX-011) in combination with docetaxel or mitoxantrone as second-line therapy in patients with metastatic castrate-resistant prostate cancer progressing after first-line docetaxel: CUOG trial P-06c. Clinical cancer research: an official journal of the American Association for Cancer Research 2011;17(17):5765-73. doi: 10.1158/1078-0432.CCR-11-0859

42. Meulenbeld HJ, Bleuse JP, Vinci EM, et al. Randomized phase II study of danusertib in patients with metastatic castration-resistant prostate cancer after docetaxel failure. BJU international 2013;111(1):44-52. doi: 10.1111/j.1464-410X.2012.11404.x

43. Droz JP, Medioni J, Chevreau C, et al. Randomized phase II study of nintedanib in metastatic castration-resistant prostate cancer postdocetaxel. Anticancer Drugs 2014;25(9):1081-8. doi: 10.1097/CAD.0000000000000131

44. Hussain M, Rathkopf D, Liu G, et al. A randomised non-comparative phase II trial of cixutumumab (IMC-A12) or ramucirumab (IMC-1121B) plus mitoxantrone and prednisone in men with metastatic docetaxel-pretreated castration-resistant prostate cancer. Eur I Cancer 2015;51(13):1714-24. doi: 10.1016/j.ejca.2015.05.019

45. Green AK, Corty RW, Wood WA, et al. Comparative effectiveness of mitoxantrone plus prednisone versus prednisone alone in metastatic castrate-resistant prostate cancer after docetaxel failure. Oncologist 2015;20(5):516-22. doi: 10.1634/theoncologist.2014-0432

46. Fizazi K, Scher HI, Molina A, et al. Abiraterone acetate for treatment of metastatic castration-resistant prostate cancer: final overall survival analysis of the COU-AA-301 randomised, double-blind, placebo-controlled phase 3 study. Lancet Oncol 2012;13(10):983-92. doi: 10.1016/S1470-2045(12)70379-0 [published Online First: 2012/09/22]

47. Ryan CJ, Smith MR, de Bono JS, et al. Abiraterone in metastatic prostate cancer without previous chemotherapy. The New England journal of medicine 2013;368(2):138-48. doi: 10.1056/NEJMoa1209096

48. Clark MJ, Harris N, Griebsch I, et al. Patient-reported outcome labeling claims and measurement approach for metastatic castration-resistant prostate cancer treatments in the United States and European Union. Health Qual Life Outcomes 2014-12:104 doi: 10.1186/s12955-014-0104-5

49. Beer TM, Armstrong AJ, Rathkopf DE, et al. Enzalutamide in metastatic prostate cancer before chemotherapy. N Engl J Med 2014;371(5):424-33. doi: 10.1056/NEJMoa1405095

50. Beer TM, Armstrong AJ, Rathkopf D, et al. Enzalutamide in Men with Chemotherapy-naive Metastatic Castration-resistant Prostate Cancer: Extended Analysis of the Phase 3 PREVAIL Study. Eur Urol 2017;71(2):151-54. doi: 10.1016/j.eururo.2016.07.032

51. Li Z, Alyamani M, Li J, et al. Redirecting abiraterone metabolism to fine-tune prostate cancer anti-androgen therapy. Nature 2016;533(7604):547-51. doi: 10.1038/nature17954 [published Online First: 2016/05/27]

52. Basch E, Reeve BB, Mitchell SA, et al. Development of the National Cancer Institute's patient-reported outcomes version of the common terminology criteria for adverse events (PRO-CTCAE). J Natl Cancer Inst 2014;106(9) doi: 10.1093/jnci/dju244 